\title{
Solar Powered Radioactive Air Monitoring Stations
}

JM Barnett

TL Gervais

LE Bisping

October 2013

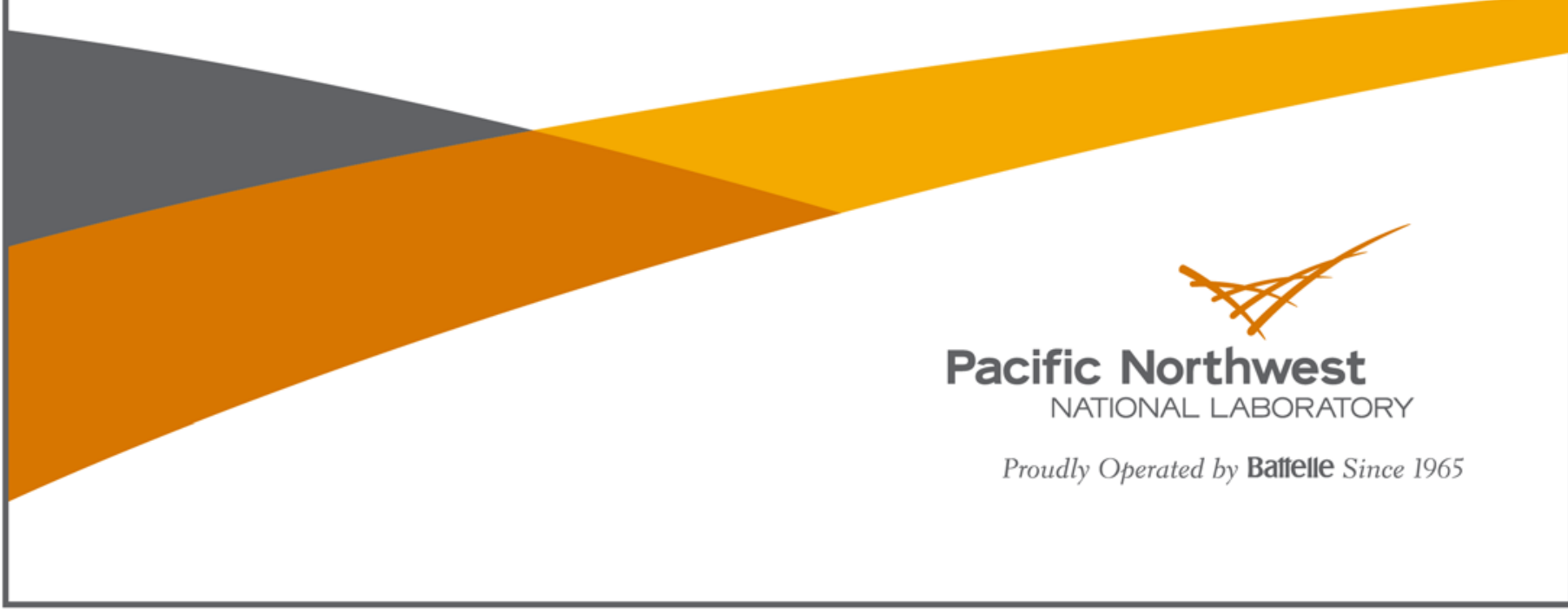




\title{
DISCLAIMER
}

This report was prepared as an account of work sponsored by an agency of the United States Government. Neither the United States Government nor any agency thereof, nor Battelle Memorial Institute, nor any of their employees, makes any warranty, express or implied, or assumes any legal liability or responsibility for the accuracy, completeness, or usefulness of any information, apparatus, product, or process disclosed, or represents that its use would not infringe privately owned rights. Reference herein to any specific commercial product, process, or service by trade name, trademark, manufacturer, or otherwise does not necessarily constitute or imply its endorsement, recommendation, or favoring by the United States Government or any agency thereof, or Battelle Memorial Institute. The views and opinions of authors expressed herein do not necessarily state or reflect those of the United States Government or any agency thereof.

\author{
PACIFIC NORTHWEST NATIONAL LABORATORY \\ operated by \\ BATTELLE \\ for the \\ UNITED STATES DEPARTMENT OF ENERGY \\ under Contract DE-AC05-76RL01830
}

Printed in the United States of America
Available to DOE and DOE contractors from the Office of Scientific and Technical Information,
P.O. Box 62, Oak Ridge, TN 37831-0062;
ph: (865) 576-8401
fax: $(865) 576-5728$
email: reports@adonis.osti.gov
Available to the public from the National Technical Information Service 5301 Shawnee Rd., Alexandria, VA 22312 ph: (800) 553-NTIS (6847)
email: orders@ntis.gov $<$ http://www.ntis.gov/about/form.aspx $>$ Online ordering: http://www.ntis.gov




\section{Solar Powered Radioactive Air Monitoring Stations}

JM Barnett

TL Gervais

LE Bisping

October 2013

Prepared for

the U.S. Department of Energy

under Contract DE-AC05-76RL01830

Pacific Northwest National Laboratory

Richland, Washington 99352 



\section{Summary}

Environmental monitoring of ambient air for radioactive material is required as stipulated in the PNNL Site radioactive air license. Sampling ambient air at identified preferred locations could not be initially accomplished because utilities were not readily available. Therefore, solar powered environmental monitoring systems were considered as a possible option. PNNL purchased two 24-V DC solar powered environmental monitoring systems which consisted of solar panels, battery banks, and sampling units. During an approximate four month performance evaluation period, the solar stations operated satisfactorily at an on-site test location. They were subsequently relocated to their preferred locations in June 2012 where they continue to function adequately under the conditions found in Richland, Washington. 



\section{Acronyms and Abbreviations}

$\begin{array}{ll}\text { cfm } & \text { cubic feet per minute } \\ \mathrm{ft}^{3} & \text { cubic feet } \\ \text { in } & \text { inch(es) } \\ \mathrm{m}^{3} & \text { cubic meters } \\ \mathrm{mm} & \text { millimeter(s) } \\ \text { PLC } & \text { programmable logic controller } \\ \text { PNNL } & \text { Pacific Northwest National Laboratory } \\ \text { RAEL } & \text { Radioactive Air Emissions License } \\ \text { RPT } & \text { Radiation Protection Technologist }\end{array}$





\section{Contents}

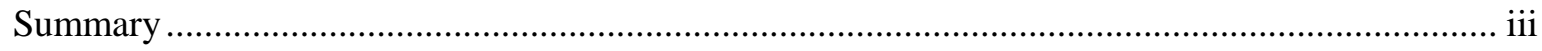

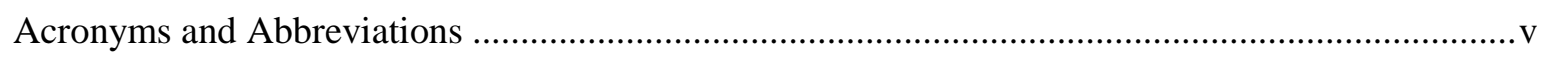

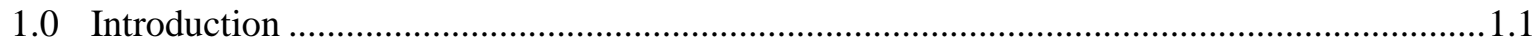

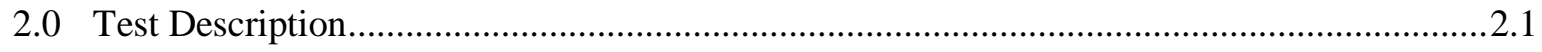

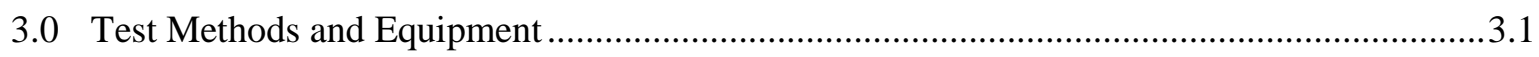

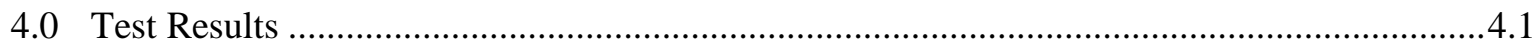

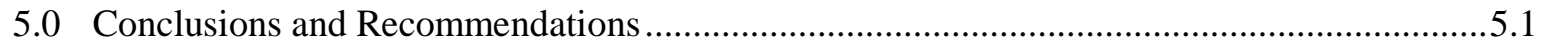

Appendix A Daily Monitoring Checklist Data ...................................................................... A.1

Appendix B GEL Laboratories Analytical Results for 2-in Sample Filters.................................... B.1

Appendix C Daily Monitoring Checklist Sample Form ............................................................ C.1

\section{Figures}

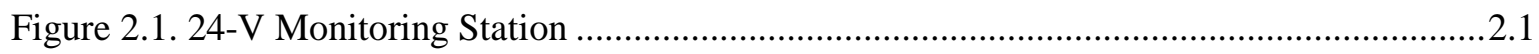

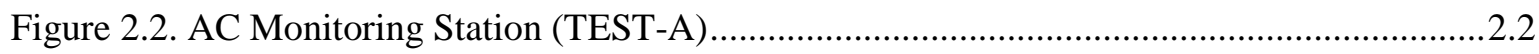

Figure 2.3. Battelle Pacific Northwest Ball Field and Test Area..................................................2.2

Figure 2.4. Permanent Locations for the Solar Monitoring Stations ............................................2.3

Figure 3.1. Solar Environmental Monitoring System with 2-in Sample Holder Exposed................3.1

Figure 4.1. Results of Gross Alpha Analysis of Particulate Air Samples .......................................4.2

Figure 4.2. Results of Gross Beta Analysis of Particulate Air Samples .......................................4.3

\section{Table}

Table 2.1. Test System Configurations ................................................................................. 2.1 



\subsection{Introduction}

Pacific Northwest National Laboratory (PNNL) currently owns and operates four ambient radioactive air environmental monitoring stations, two of which are solar powered. These stations are operated to comply with the PNNL Site Radioactive Air Emissions License-05 (RAEL-05), which requires the monitoring of ambient air concentrations to confirm low emissions of radionuclides in air. In 2010, a Data Quality Objectives report ${ }^{1}$ that supported radiological air emissions from the PNNL Site located in Richland, WA identified preferred ambient air sampling locations; however, utilities were not readily available at two locations on the site boundary. Therefore, solar powered environmental monitoring systems were considered as a possible option. PNNL purchased two 24-V DC solar powered environmental monitoring stations which consisted of solar panels, battery banks, and sampling units. During an approximate 4month performance evaluation period, the solar stations operated satisfactorily at an on-site test location. The stations were subsequently relocated to their preferred locations in June 2012, where they continue to function normally.

\footnotetext{
${ }^{1}$ Barnett, JM, KM Meier, SF Snyder, BG Fritz, TM Poston, and K Rhoads. 2010. "Data Quality Objectives Supporting Radiological Air Emissions Monitoring for the PNNL Site,” PNNL-19427. Pacific Northwest National Laboratory, Richland, Washington.
} 



\subsection{Test Description}

Two 24-V DC solar powered environmental monitoring stations (model CF-5624WR) were procured from HI-Q Environmental Products Company (7386 Trade St, San Diego, CA 92121) and operated to evaluate system performance (Figure 2.1). TEST-B system was configured with a 2-inch (in) sample filter, and the TEST-C system was configured with a 4-in sample filter (refer to Table 2.1 for test configurations). TEST-C was reconfigured and renamed TEST-D later in the test period because the 2-in filter would work for the application. TEST-D is an identical 2-in sample filter arrangement as found in TEST-B. To provide comparative sampling results, an AC sampling system was used that consisted of a small housed station with an air volume totalizer, flow controller, and a vacuum pump. This specific setup was identical to the existing AC monitoring stations placed at the same location (Figure 2.2) and is identified as TEST-A in Table 2.1. These test stations were placed approximately 100 feet west of the Battelle Pacific Northwest ball fields (Figure 2.3) in an area where the solar panels would experience full sun when available.

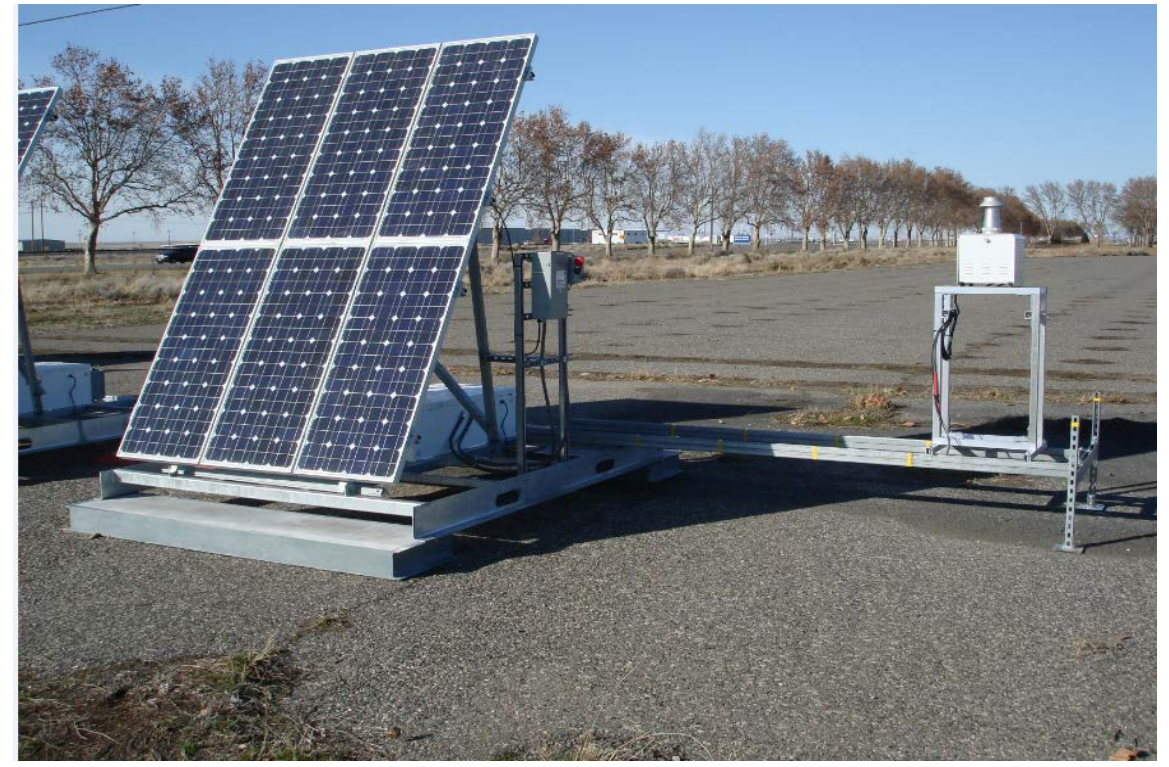

Figure 2.1. 24-V Monitoring Station

Table 2.1. Test System Configurations

\begin{tabular}{clc}
\hline Test Name & \multicolumn{1}{c}{ Power Source } & Filter Dimension (in) \\
\hline TEST-A & AC (120 V) & 2 \\
TEST-B & DC (24 V solar battery array) & 2 \\
TEST-C & DC (24 V solar battery array) & 4 \\
TEST-D & DC (24 V solar battery array) & 2 \\
\hline
\end{tabular}




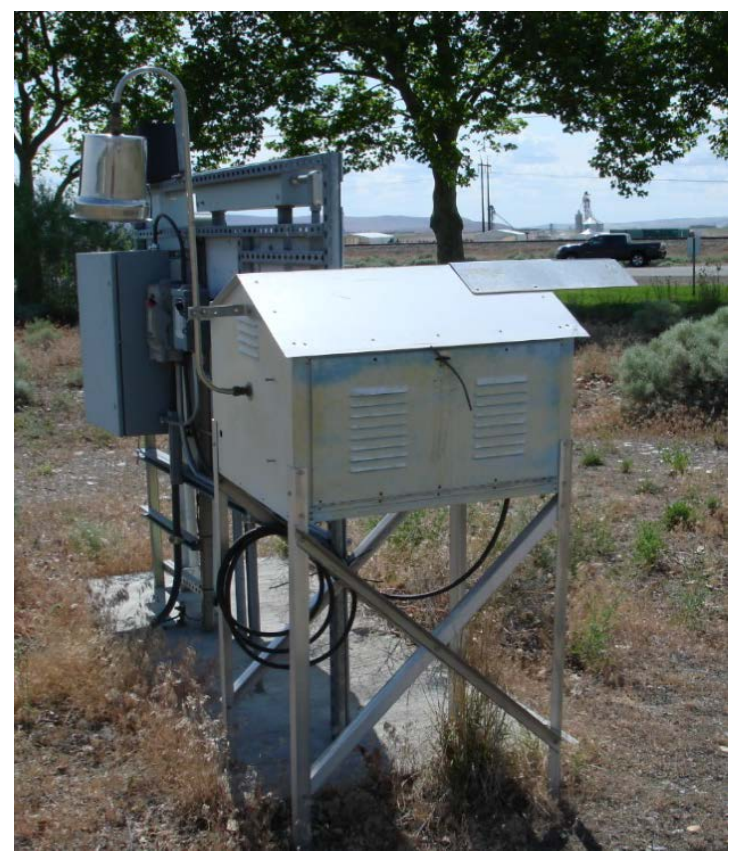

Figure 2.2. AC Monitoring Station (TEST-A)

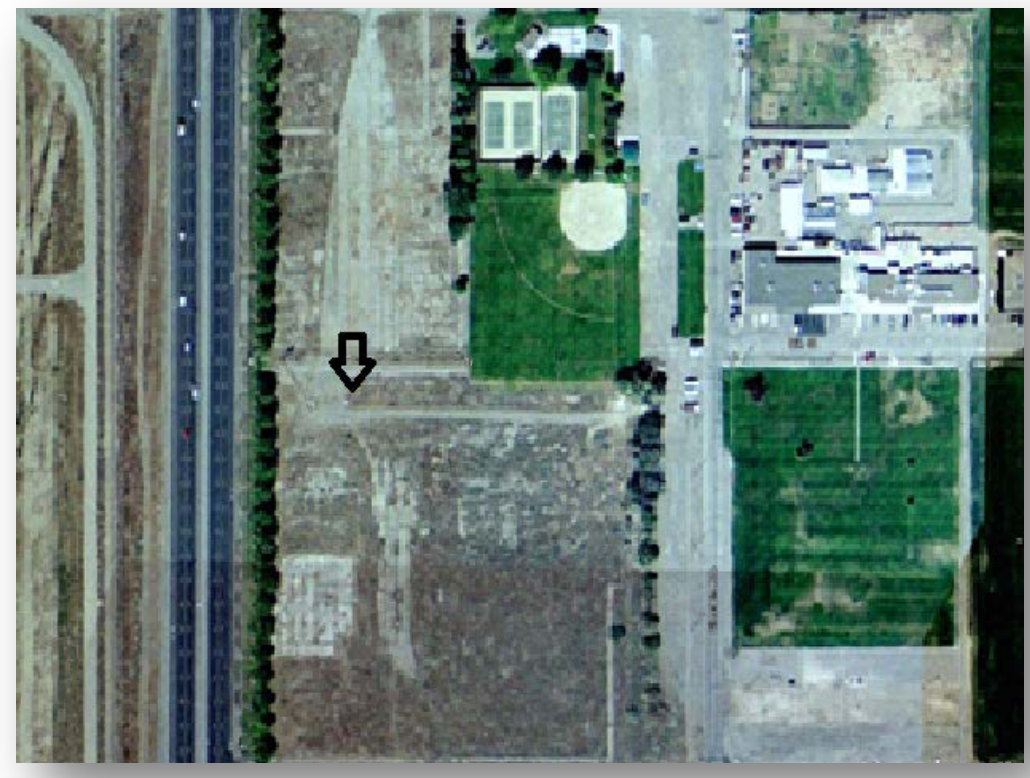

Figure 2.3. Battelle Pacific Northwest Ball Field and Test Area

Solar sampler testing began on February 22, 2012. On weekdays, Radiation Protection Technologist (RPT) staff would document system status and record data for each environmental monitoring station (Appendix A). Samples for each station were collected bi-weekly, and collection coincided with the AC 
monitoring stations. The 2-in sample filters collected from TEST-A, TEST-B, and TEST-D were submitted for analyses; however, the 4-in filters collected from TEST-C were archived and not analyzed because the filter size is larger than the standard 2 in detector size. The objectives of the testing were to determine if the sampler was able to maintain a nominal sample flow of 1.5 cubic feet per minute (cfm), to demonstrate if the environmental monitoring system would work under all weather conditions, to determine if the power supplied by the solar panels would be adequate, and to determine if the analytical results would be equivalent to the AC powered systems.

Following the test period, the two solar powered monitoring stations were placed in their permanent locations as indicated in Figure 2.4.

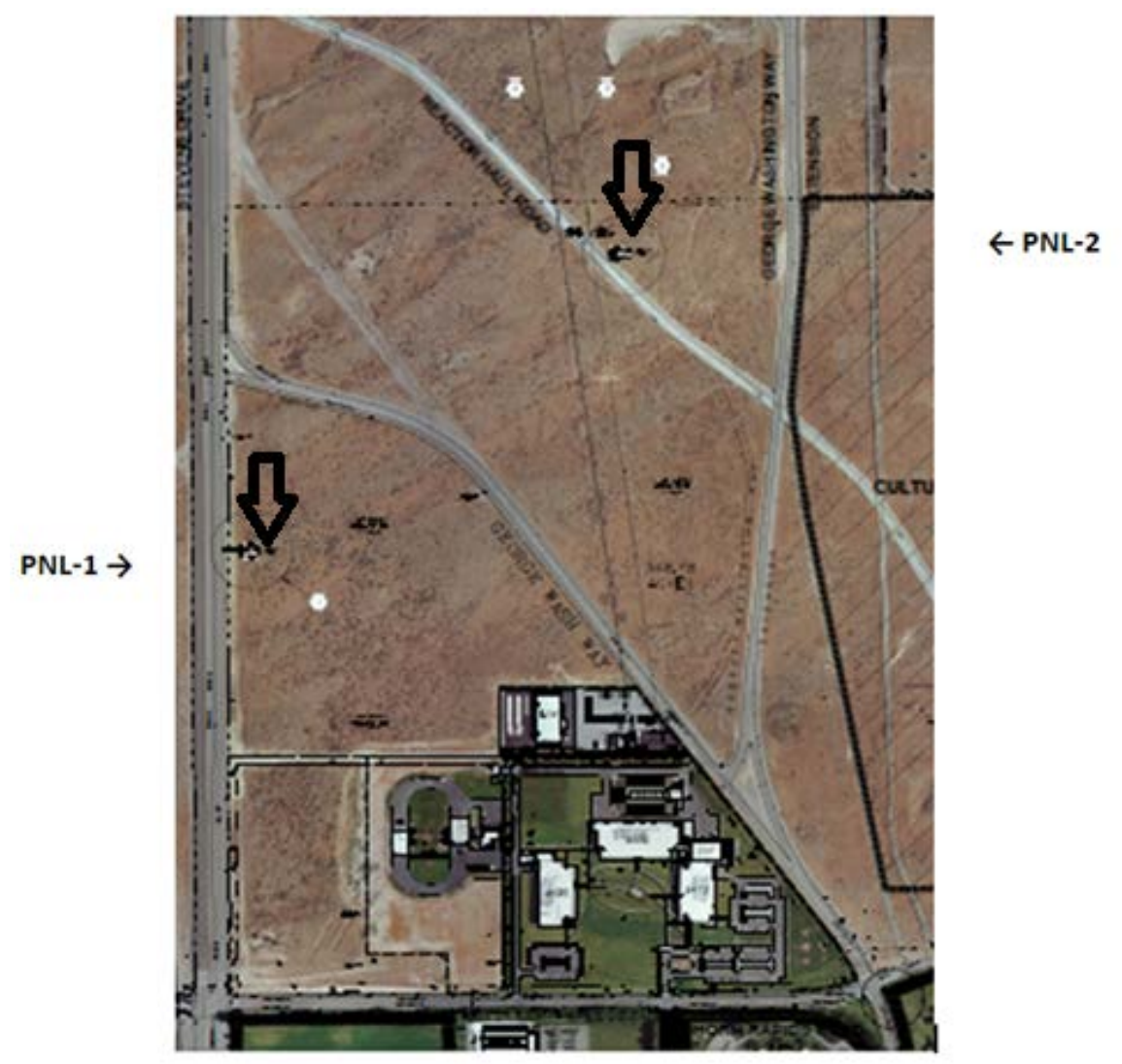

Figure 2.4. Permanent Locations for the Solar Monitoring Stations 



\subsection{Test Methods and Equipment}

The main components of the tested 24-V DC sampling systems included the sampling unit (CF-5624D brushless, automatic flow control, DC-powered, low-volume air sampler), solar panels, and battery banks. If any of these components were inadequate or failed, the system as a whole would not collect the sample as required. The two sample systems tested (referred to as TEST-B and TEST-C) were set up west of the ball fields. TEST-B was set up with the 2-in filter head, and TEST-C was outfitted with the 4-in filter head. Though the 2-in is the standard filter size used for sampling systems, the 4-in filter head was evaluated as an alternative sample method in the event that the 2-in filter could not pull the requisite volume during the 2-week sample period. Midway into the test it was apparent that the 2-in filter could be used and the second solar array was reconfigured as TEST-D with a 2-in filter head.

Figure 3.1 depicts the 2-in filter holder of an environmental monitoring system. The rain cap that would normally be situated over the filter holder and connector was set aside. Air is pulled through the filter head assembly, which contains a 47-millimeter (mm) glass fiber filter, at a desired rate of $1.5 \mathrm{cfm}$.

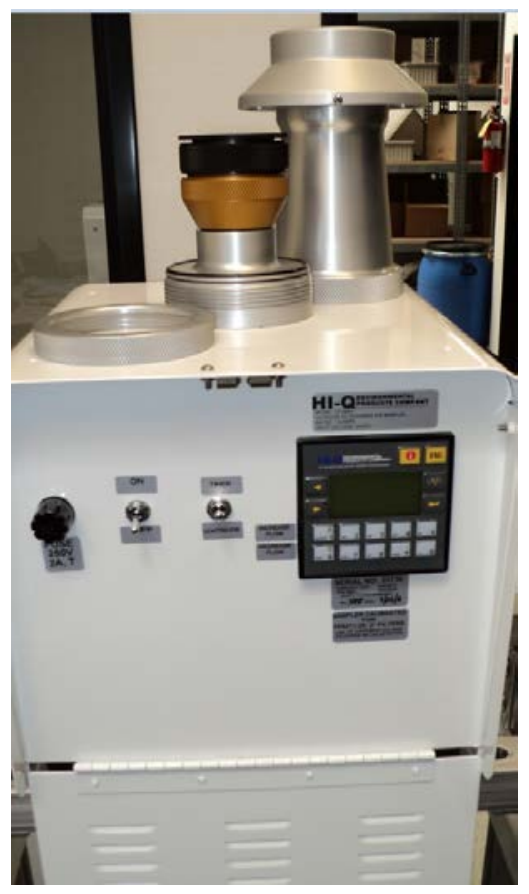

Figure 3.1. Solar Environmental Monitoring System with 2-in Sample Holder Exposed

An AC environmental monitoring system identified as TEST-A commenced on January 17, 2012 as a comparative sampler for validating analytical results. TEST-A is an AC system identical to the existing environmental monitoring systems and consists of a small housed station containing an air volume totalizer, vacuum pump, and flow controller.

The solar panels of the environmental monitoring system were positioned to face the south to optimize solar influence. The stations were operated under the same conditions expected to be found in the desired sample locations. The sampling units came pre-calibrated and programmed to a sample rate of $1.5 \mathrm{cfm}$. A detailed step-by-step explanation of the process and operation of the environmental monitoring systems 
can be found in the test plan Evaluation and Operation of 24 V DC Powered Environmental Monitoring Stations (EMPT-AIR-001). The solar environmental monitoring system testing began on February 22, 2012. The RPT collected the following data on scheduled workdays (Appendix A):

- date

- time

- flow rate

- total flow

- elapsed time

- battery condition

- environmental conditions.

Issues with monitoring system performance were addressed as soon as practical after the problem was discovered/identified. Sample filter collection was performed bi-weekly. The 2-in sample filters were sent to GEL Laboratories for gross alpha/gross beta analyses. GEL retained the filters for radioisotope specific composite analyses. The 4-in TEST-C filters were not analyzed, but were all archived locally for possible later analyses.

The Test Plan outlined the basic qualification criteria pertaining to the HI-Q system. The specifications necessary for the solar powered environmental monitoring systems to qualify as appropriate for sampling are described in section 4.0.

The AC monitoring system (TEST-A) was operated and sampled using procedure Air Particulate Sampling and Routine Maintenance of Environmental Monitoring Stations for the PNNL Site (EPRPAIR-029). Sample information from both the DC and AC systems is included with current sampling documentation collected by RPT staff. 


\subsection{Test Results}

The solar powered environmental monitoring systems were tested over an approximate 4-month period (February 22, 2012 through June 27, 2012), during which time the environmental conditions were also evaluated. Appendix A provides data collected by RPT staff over the test period and information about the operational feasibility of system usage. Data evaluation was useful in optimizing the system and verifying operational qualification. A detailed discussion about each specification is included in the discussion below.

Specification 1: Sample Flow - Maintain flow through the sample head at $1.5 \mathrm{cfm}$ without variation throughout each 2-week sample period due to diurnal changes or other environmental conditions.

Maintaining a sample flow of $1.5 \mathrm{cfm}$ is important because at this flow rate, the sample volume meets the minimum detection and sample requirements. If the 1.5-cfm flow cannot be sustained, then the required volume of sample required may not be attained resulting in a "no-sample." Information received from daily flow checks showed that instantaneous flow rates fluctuated between 1.4 and $1.5 \mathrm{cfm}$. The control unit of the system constantly monitors the flow rate and adjusts the sample rate as necessary to maintain the pre-set flow rate of $1.5 \mathrm{cfm}$, and the system is designed not to exceed the pre-set flow rate value. Sample flow rate averages over the observed 2-week operating periods ranged from 1.489 to $1.497 \mathrm{cfm}$ for the sample periods in which the unit was pre-set to $1.5 \mathrm{cfm}$. On March 22, 2012, the pre-set flow rate value increased to $1.6 \mathrm{cfm}$; for this setting, the flow rate averages over the 2-week operating period were between 1.594 and $1.597 \mathrm{cfm}$.

Specification 2: Blower Operation - The blower on the environmental monitoring system must operate under both hot and cold weather extremes. The blower must have the capacity to continue sampling at $1.5 \mathrm{cfm}$ even if the filter contains an accumulation of dust or ice particles.

Sample collection depends on the ability of the air sampler to operate in adverse weather conditions. Over the time period evaluated (February 22, 2012 through June 27, 2012), the following environmental conditions existed: low-light conditions, temperatures below $32^{\circ} \mathrm{F}$ and greater than $90^{\circ} \mathrm{F}$, overcast conditions, rain, and dust. The environmental conditions experienced did not adversely affect the environmental monitoring systems. Once it was apparent that the 2-in filter system operated normally (e.g., it did not experience sample failure or excessive particulate buildup during the 2-week sample period) the 4-in filter system (TEST-C) was replaced with a 2-in filter system and renamed TEST-D.

Blower/sample unit failure did occur for reasons other than environmental conditions. Both conditions involved equipment failure. TEST-B had a system malfunction which involved a power disruption during low light conditions. On May 18, 2012 and June 4, 2012, the environmental monitoring system shut down due to the lack of power. Troubleshooting showed the power controller inside the battery box overheated causing the controller to operate unpredictably. Corrective action included ordering additional power controllers and replacing the failed power controller.

TEST-C ceased operation on March 21, 2012 and again on April 13, 2012. Both instances involved problems with the programmable logic controller (PLC) on the system. The first time the blower stopped, the issue was resolved by shutting down and re-booting the system. The second time the blower went down, the system would not re-boot, so the unit was returned to the vendor for service. TEST-C operated 
normally with the new blower installed. Despite these malfunctions, the system passed the specification because the sample flow rate could be maintained.

Specification 3: Solar Panel Charging Ability and Battery Life - The charging system and battery capacity must be adequate to run the monitoring system as desired for extended periods of low daylight conditions, as overcast and foggy conditions may extend for multiple weeks at a time during the winter.

Low-light conditions experienced during winter months and during heavy overcast weather impacts the ability of the solar powered system to charge the battery array and maintain a steady power source to the environmental monitoring system. To obtain an accurate battery charge reading, RPTs made daily checks before 8:00 am when possible. During the test period, the battery arrays remained charged and functional. The environmental monitoring system ceases operation when the battery array charge drops below $20 \mathrm{~V}$. Overall, the solar charging ability and battery capacity were adequate under the environmental conditions experienced during the test period; $23 \mathrm{~V}$ was the lowest battery charge reading

Specification 4: Uniform Analytical Results - Collect analytical samples from the HI-Q systems equivalent to the AC powered systems.

The 2-in particulate sample filters for TEST-A, TEST-B, and TEST-D (TEST-D was started April 18, 2012) were sent off for gross alpha and gross beta analysis after each 2-week sample period. The 2-in sample filters were analyzed at GEL Laboratories (2040 Savage Rd, Charleston, SC 29407) for nondestructive gross alpha and gross beta analysis. Comparative plots of the gross alpha and gross beta analytical results are shown in Figure 4.1 and Figure 4.2 and show a relative degree of similarity. The samples were held and analytical composites for select nuclides were made at the end of June 2012. Data composite results are also similar in that results were below detection limits for most radionuclides for all systems and the few results above detection limits had similar values for all systems (see Appendix B).

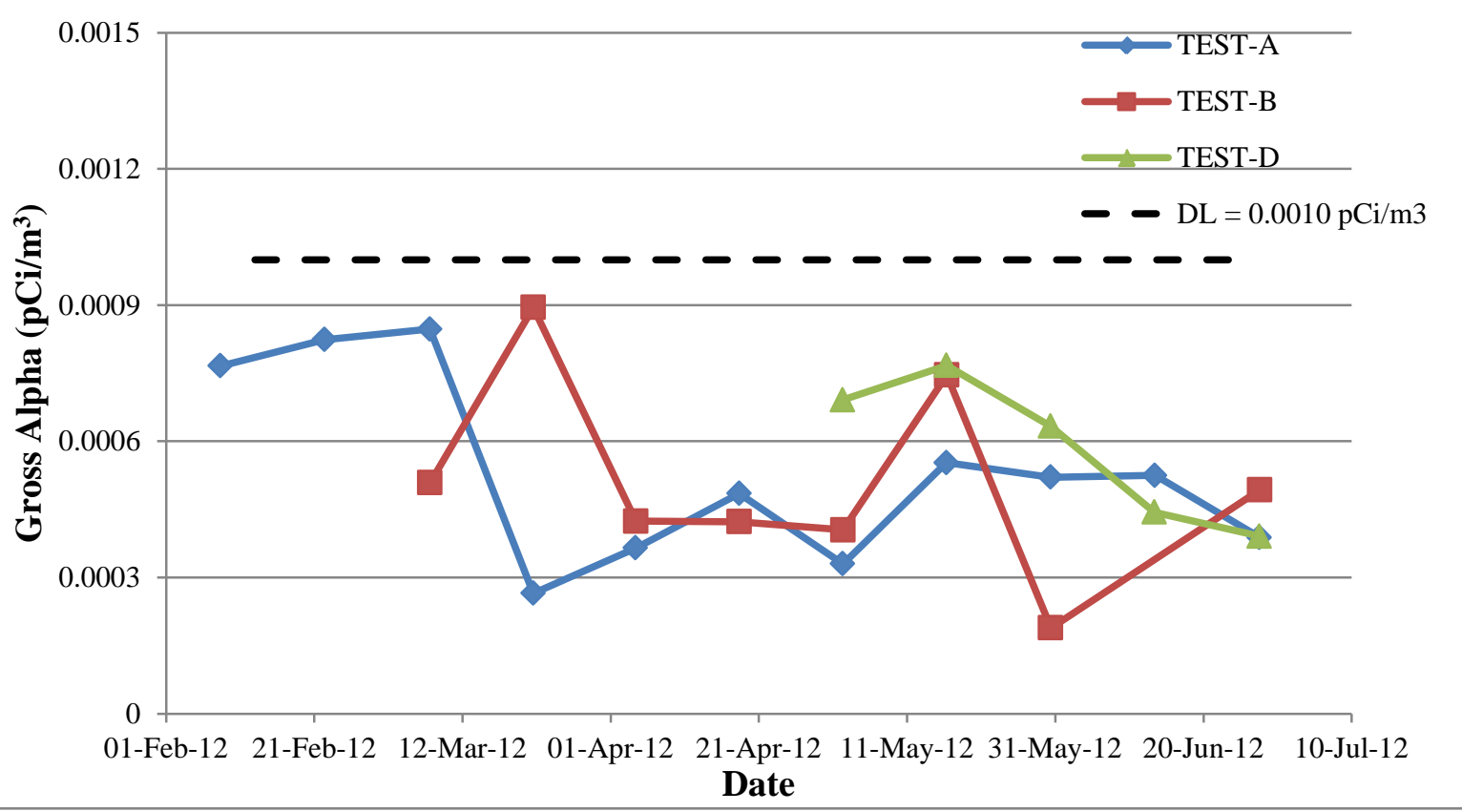

Figure 4.1. Results of Gross Alpha Analysis of Particulate Air Samples (Detection Limit is $0.0010 \mathrm{pCi} / \mathrm{m}^{3}$ ) 


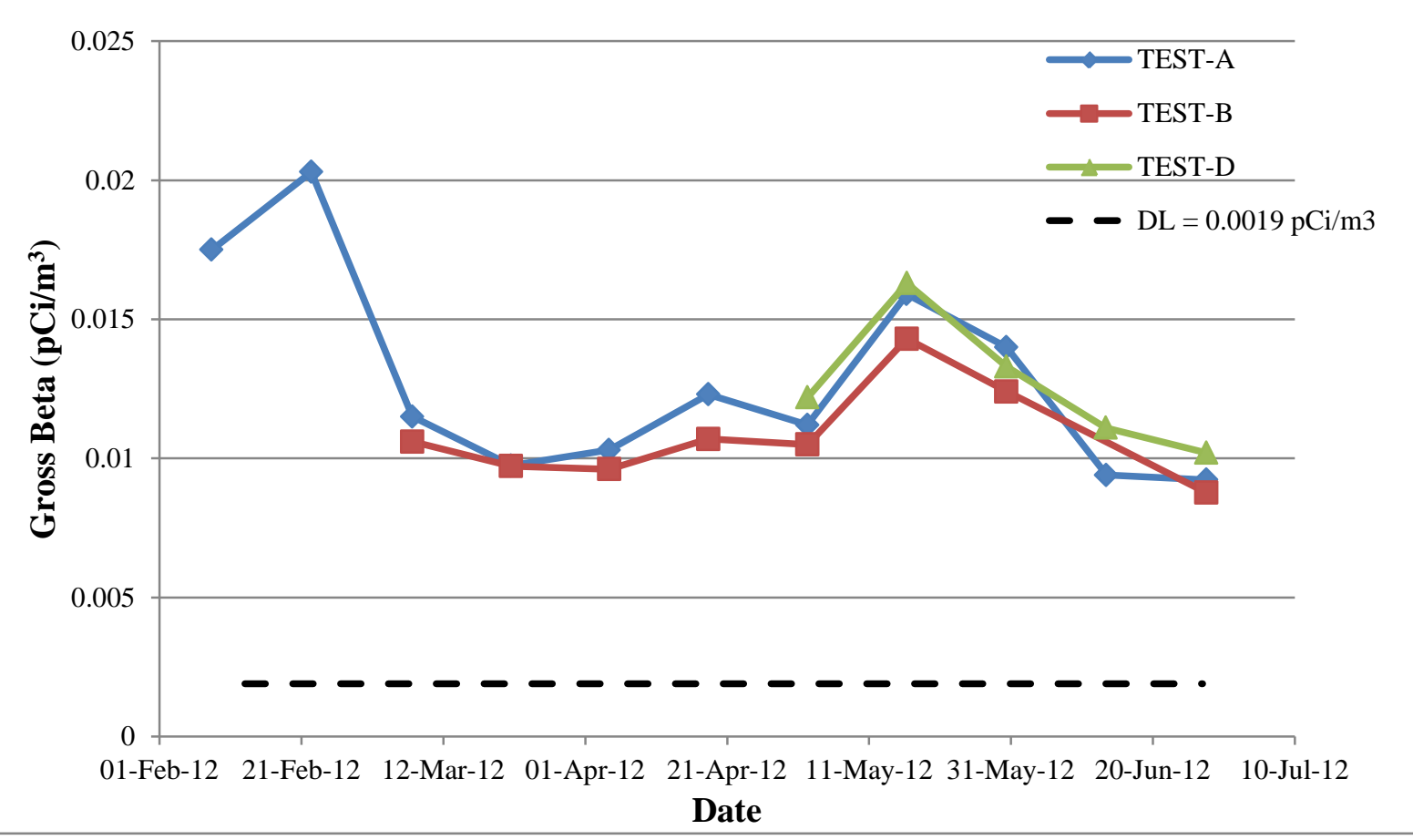

Figure 4.2. Results of Gross Beta Analysis of Particulate Air Samples (Detection Limit is 0.0019 pCi/m³) 



\subsection{Conclusions and Recommendations}

For the evaluation period, the solar powered monitoring stations meet the established qualification criteria. Results show the solar powered system is a feasible alternative to current AC sampling systems. Sample flows were maintained, and the required air volume sampled was met for each 2-week sample period. The blower operation manages the environmental conditions experienced; however, the solar environmental monitoring stations are more susceptible to failure from the many different components, program software, and electrical connections. Replacement components should be available to maintain the $85 \%$ or greater operability factor. The charging ability of the solar panels and battery capacity appear to be sufficient for the power usage required at the $1.6 \mathrm{cfm}$ sample rate. Finally, the test samples analyzed for gross alpha/gross beta indicate similar results.

Based on the tests performed and observations made, the solar powered monitoring stations were deployed to their permanent locations in June 2012 as a viable environmental monitoring method to comply with the PNNL Site RAEL-05. 

Appendix A

\section{Daily Monitoring Checklist}





\section{Appendix A}

\section{Daily Monitoring Checklist Data}

\begin{tabular}{|c|c|c|c|c|c|c|c|c|c|c|}
\hline Date & $\begin{array}{c}\text { Monitoring } \\
\text { System }\end{array}$ & RPT & Time & $\begin{array}{l}\text { Flow } \\
\text { Rate } \\
(\mathbf{c f m}) \\
\end{array}$ & $\begin{array}{c}\text { End } \\
\text { Volume } \\
\left(\mathbf{m}^{3}\right) \\
\end{array}$ & $\begin{array}{c}\text { Total } \\
\text { Flow } \\
\left(\mathbf{f t}^{3}\right) \\
\end{array}$ & $\begin{array}{c}\text { Total } \\
\text { Flow }\left(\mathbf{f t}^{3}\right) \\
\text { converted } \\
\text { to } \mathbf{~ m}^{3} \\
\end{array}$ & $\begin{array}{c}\text { Elapsed } \\
\text { Time } \\
\text { (Hrs) } \\
\end{array}$ & $\begin{array}{c}\text { Battery } \\
\text { Condition } \\
\text { (V) }\end{array}$ & $\begin{array}{c}\text { Environmental } \\
\text { Conditions }\end{array}$ \\
\hline \multirow{3}{*}{ 22-Feb-12 } & TEST-A & DLM & 0915 & $--{ }^{(1)}$ & 8283 & - -- $^{(1)}$ & $--^{(1)}$ & $--{ }^{(1)}$ & $--{ }^{(1)}$ & (2) \\
\hline & TEST-B & DLM & 0915 & 1.5 & --- & 0 & 0 & 0 & 27 & (2) \\
\hline & TEST-C & DLM & 0915 & 1.5 & --- & 0 & 0 & 0 & 27 & (2) \\
\hline \multirow{3}{*}{ 23-Feb-12 } & TEST-A & DLM & 1345 & --- & 8344 & --- & --- & --- & --- & (2) \\
\hline & TEST-B & DLM & 1345 & 1.4 & --- & 2340 & 66 & 26.1 & 28 & (2) \\
\hline & TEST-C & DLM & 1345 & 1.4 & --- & 2349 & 66 & 26.2 & 28 & (2) \\
\hline \multirow{3}{*}{ 24-Feb-12 } & TEST-A & DLM & 1015 & --- & 8394 & --- & --- & --- & --- & (2) \\
\hline & TEST-B & DLM & 1015 & 1.4 & --- & 4185 & 118 & 46.6 & 27 & (2) \\
\hline & TEST-C & DLM & 1015 & 1.4 & --- & 4193 & 119 & 46.7 & 26 & (2) \\
\hline \multirow{3}{*}{ 27-Feb-12 } & TEST-A & SKM & 1310 & --- & 8573 & --- & --- & --- & --- & (2) \\
\hline & TEST-B & SKM & 1310 & 1.5 & --- & 10907 & 309 & 121.4 & 27 & (2) \\
\hline & TEST-C & SKM & 1310 & 1.5 & --- & 10909 & 309 & 121.5 & 27 & (2) \\
\hline \multirow{3}{*}{ 28-Feb-12 } & TEST-A & DLM & 0930 & --- & 8621 & --- & --- & --- & --- & (2) \\
\hline & TEST-B & DLM & 0930 & 1.4 & --- & 12735 & 360 & 141.8 & 28 & (2) \\
\hline & TEST-C & DLM & 0930 & 1.5 & --- & 12735 & 360 & 141.8 & 27 & (2) \\
\hline \multirow{3}{*}{ 29-Feb-12 } & TEST-A & DLM & 0855 & --- & 8677 & --- & --- & --- & --- & (2) \\
\hline & TEST-B & DLM & 0855 & 1.5 & --- & 14836 & 420 & 165.1 & 29 & (2) \\
\hline & TEST-C & DLM & 0855 & 1.5 & --- & 14840 & 420 & 165.3 & 27 & (2) \\
\hline \multirow{3}{*}{ 1-Mar-12 } & TEST-A & FG & 1315 & --- & 8745 & --- & --- & --- & --- & (2) \\
\hline & TEST-B & FG & 1315 & 1.5 & --- & 17371 & 492 & 193.3 & 27 & (2) \\
\hline & TEST-C & FG & 1315 & 1.5 & --- & 17373 & 492 & 193.5 & 27 & (2) \\
\hline \multirow{3}{*}{ 2-Mar-12 } & TEST-A & FG & 1430 & --- & 8807 & --- & --- & --- & --- & (2) \\
\hline & TEST-B & FG & 1430 & 1.5 & --- & 19649 & 556 & 218.7 & 27 & (2) \\
\hline & TEST-C & FG & 1430 & 1.5 & --- & 19648 & 556 & 218.8 & 27 & (2) \\
\hline \multirow{3}{*}{ 5-Mar-12 } & TEST-A & FG & 1130 & --- & 8973 & --- & --- & --- & --- & (2) \\
\hline & TEST-B & FG & 1130 & 1.5 & --- & 25849 & 732 & 287.6 & 28 & (2) \\
\hline & TEST-C & FG & 1130 & 1.4 & --- & 25840 & 731 & 287.7 & 27 & (2) \\
\hline \multirow{3}{*}{ 6-Mar-12 } & TEST-A & FG & 0930 & --- & 9024 & --- & --- & --- & --- & (2) \\
\hline & TEST-B & FG & 0930 & 1.5 & --- & 27802 & 787 & 309.4 & 28 & (2) \\
\hline & TEST-C & FG & 0930 & 1.5 & --- & 27795 & 787 & 309.5 & 27 & (2) \\
\hline \multirow{3}{*}{ 7-Mar-12 } & TEST-A & FG & 1445 & --- & 9095 & --- & --- & --- & --- & Sunny \\
\hline & TEST-B & FG & 1500 & 1.5 & --- & 30465 & 862 & 339 & 27 & Sunny \\
\hline & TEST-C & FG & 1545 & 1.5 & --- & 30514 & 864 & 339.8 & 27 & Sunny \\
\hline \multirow{3}{*}{ 8-Mar-12 } & TEST-A & FG & 1300 & --- & 9152 & --- & --- & --- & --- & Sunny \\
\hline & TEST-B & FG & 1300 & 1.5 & --- & 1923 & 54 & 21.4 & 27 & Sunny \\
\hline & TEST-C & FG & 1300 & 1.5 & --- & 1898 & 54 & 21.8 & 27 & Sunny \\
\hline \multirow{3}{*}{ 9-Mar-12 } & TEST-A & FG & 0915 & --- & 9205 & --- & --- & --- & --- & Sunny \\
\hline & TEST-B & FG & 0915 & 1.5 & --- & 3745 & 106 & 41.7 & 28 & Sunny \\
\hline & TEST-C & FG & 0915 & 1.5 & --- & 3713 & 105 & 41.4 & 27 & Sunny \\
\hline
\end{tabular}




\begin{tabular}{|c|c|c|c|c|c|c|c|c|c|c|}
\hline Date & $\begin{array}{l}\text { Monitoring } \\
\text { System }\end{array}$ & RPT & Time & $\begin{array}{l}\text { Flow } \\
\text { Rate } \\
\text { (cfm) }\end{array}$ & $\begin{array}{c}\text { End } \\
\text { Volume } \\
\left(\mathbf{m}^{3}\right)\end{array}$ & $\begin{array}{c}\text { Total } \\
\text { Flow } \\
\left(\mathbf{f t}^{3}\right)\end{array}$ & $\begin{array}{c}\text { Total } \\
\text { Flow }\left(\mathrm{ft}^{3}\right) \\
\text { converted } \\
\text { to } \mathbf{~ m}^{3}\end{array}$ & $\begin{array}{c}\text { Elapsed } \\
\text { Time } \\
\text { (Hrs) }\end{array}$ & $\begin{array}{c}\text { Battery } \\
\text { Condition } \\
\text { (V) }\end{array}$ & $\begin{array}{c}\text { Environmental } \\
\text { Conditions }\end{array}$ \\
\hline \multirow{3}{*}{ 12-Mar-12 } & TEST-A & FG & 1400 & --- & 9397 & --- & --- & --- & --- & Cloudy \\
\hline & TEST-B & FG & 1400 & 1.5 & --- & 10552 & 299 & 117.4 & 28 & Cloudy \\
\hline & TEST-C & FG & 1400 & 1.4 & --- & 10510 & 297 & 117.1 & 27 & Cloudy \\
\hline \multirow{3}{*}{ 13-Mar-12 } & TEST-A & FG & 1400 & --- & 9457 & --- & --- & --- & --- & Cloudy \\
\hline & TEST-B & FG & 1400 & 1.5 & --- & 12695 & 359 & 141.4 & 28 & Cloudy \\
\hline & TEST-C & FG & 1400 & 1.5 & --- & 12668 & 359 & 141.1 & 27 & Cloudy \\
\hline \multirow{3}{*}{ 14-Mar-12 } & TEST-A & FG & 0800 & --- & 9503 & --- & --- & --- & --- & Cloudy \\
\hline & TEST-B & FG & 0800 & 1.5 & --- & 14303 & 405 & 159.3 & 26 & Cloudy \\
\hline & TEST-C & FG & 0800 & 1.5 & --- & 14273 & 404 & 159 & 26 & Cloudy \\
\hline \multirow{3}{*}{ 15-Mar-12 } & TEST-A & FG & 1230 & --- & 9574 & --- & --- & --- & --- & Overcast \\
\hline & TEST-B & FG & 1230 & 1.5 & --- & 16835 & 476 & 187.8 & 27 & Overcast \\
\hline & TEST-C & FG & 1230 & 1.5 & --- & 16836 & 476 & 187.6 & 27 & Overcast \\
\hline \multirow{3}{*}{ 16-Mar-12 } & TEST-A & FG & 1245 & --- & 9636 & --- & --- & --- & --- & Sunny \\
\hline & TEST-B & FG & 1245 & 1.5 & --- & 18969 & 537 & 212 & 27 & Sunny \\
\hline & TEST-C & FG & 1245 & 1.5 & --- & 19005 & 538 & 211.7 & 27 & Sunny \\
\hline \multirow{3}{*}{ 19-Mar-12 } & TEST-A & FG & 1000 & --- & 9810 & --- & --- & --- & --- & Sunny \\
\hline & TEST-B & FG & 1000 & 1.5 & --- & 25147 & 712 & 281.2 & 28 & Sunny \\
\hline & TEST-C & FG & 1000 & 1.5 & --- & 25217 & 714 & 280.9 & 27 & Sunny \\
\hline \multirow{3}{*}{ 20-Mar-12 } & TEST-A & FG & 0800 & --- & 9868 & --- & --- & --- & --- & Partly Overcast \\
\hline & TEST-B $^{(3)}$ & FG & 0800 & 1.4 & --- & 27104 & 767 & 303.2 & 25 & Partly Overcast \\
\hline & TEST-C ${ }^{(3)}$ & FG & 0800 & 1.4 & --- & 27192 & 770 & 302.9 & 25 & Partly Overcast \\
\hline \multirow{3}{*}{ 21-Mar-12 } & TEST-A & FG & 1315 & --- & 9944 & --- & --- & --- & --- & Overcast Raining \\
\hline & TEST-B & FG & 1330 & 1.5 & --- & 29799 & 843 & 332.6 & 27 & Overcast Raining \\
\hline & TEST-C & FG & 1400 & 1.5 & --- & 29129 & 824 & 323.1 & 27 & Overcast Raining \\
\hline \multirow{3}{*}{ 22-Mar-12 } & TEST-A & FG & 0745 & --- & 9987 & --- & --- & --- & --- & Partly Overcast \\
\hline & TEST-B & FG & 0745 & 1.5 & --- & 1715 & 49 & 18 & 25 & Partly Overcast \\
\hline & TEST-C & FG & 0745 & 1.5 & --- & 1647 & 47 & 17.2 & 25 & Partly Overcast \\
\hline \multirow{3}{*}{ 23-Mar-12 } & TEST-A & FG & 0600 & --- & 42 & --- & --- & --- & --- & Dark \\
\hline & TEST-B & FG & 0600 & 1.6 & --- & 3842 & 109 & 40.2 & 25 & Dark \\
\hline & TEST-C & FG & 0600 & 1.6 & --- & 3776 & 107 & 39.4 & 25 & Dark \\
\hline \multirow{3}{*}{ 26-Mar-12 } & TEST-A & FG & 0830 & --- & 228 & --- & --- & --- & --- & Overcast \\
\hline & TEST-B & FG & 0830 & 1.5 & --- & 10964 & 310 & 114.6 & 26 & Overcast \\
\hline & TEST-C & FG & 0830 & 1.6 & --- & 10898 & 308 & 113.8 & 26 & Overcast \\
\hline \multirow{3}{*}{ 27-Mar-12 } & TEST-A & FG & 0730 & --- & 285 & --- & --- & --- & --- & Overcast Raining \\
\hline & TEST-B & FG & 0730 & 1.6 & --- & 13150 & 372 & 137.5 & 25 & Overcast Raining \\
\hline & TEST-C & FG & 0730 & 1.6 & --- & 10392 & 294 & 136.7 & 25 & Overcast Raining \\
\hline \multirow{3}{*}{ 28-Mar-12 } & TEST-A & FG & 0730 & --- & 345 & --- & --- & --- & --- & Overcast Raining \\
\hline & TEST-B & FG & 0730 & 1.6 & --- & 15459 & 437 & 161.6 & 25 & Overcast Raining \\
\hline & TEST-C & FG & 0730 & 1.6 & --- & 15400 & 436 & 160.7 & 25 & Overcast Raining \\
\hline \multirow{3}{*}{ 29-Mar-12 } & TEST-A & FG & 0830 & --- & 407 & --- & --- & --- & --- & Partly Cloudy \\
\hline & TEST-B & FG & 0830 & 1.5 & --- & 17841 & 505 & 186.5 & 26 & Partly Cloudy \\
\hline & TEST-C & FG & 0830 & 1.5 & --- & 17783 & 503 & 185.6 & 26 & Partly Cloudy \\
\hline \multirow{3}{*}{ 30-Mar-12 } & TEST-A & FG & 1100 & --- & 473 & --- & --- & --- & --- & Overcast Raining \\
\hline & TEST-B & FG & 1100 & 1.6 & --- & 20392 & 577 & 213.2 & 28 & Overcast Raining \\
\hline & TEST-C & FG & 1100 & 1.5 & --- & 20344 & 576 & 212.4 & 28 & Overcast Raining \\
\hline
\end{tabular}




\begin{tabular}{|c|c|c|c|c|c|c|c|c|c|c|}
\hline Date & $\begin{array}{c}\text { Monitoring } \\
\text { System } \\
\end{array}$ & RPT & Time & $\begin{array}{l}\text { Flow } \\
\text { Rate } \\
\text { (cfm) } \\
\end{array}$ & $\begin{array}{c}\text { End } \\
\text { Volume } \\
\left(\mathbf{m}^{3}\right) \\
\end{array}$ & $\begin{array}{c}\text { Total } \\
\text { Flow } \\
\left(\mathbf{f t t}^{3}\right) \\
\end{array}$ & $\begin{array}{c}\text { Total } \\
\text { Flow }\left(\mathbf{f t}^{3}\right) \\
\text { converted } \\
\text { to } \mathbf{~ m}^{3} \\
\end{array}$ & $\begin{array}{c}\text { Elapsed } \\
\text { Time } \\
\text { (Hrs) }\end{array}$ & $\begin{array}{c}\text { Battery } \\
\text { Condition } \\
\text { (V) }\end{array}$ & $\begin{array}{c}\text { Environmental } \\
\text { Conditions }\end{array}$ \\
\hline \multirow{3}{*}{ 2-Apr-12 } & TEST-A & SKM & 0930 & --- & 651 & -- & --- & --- & -- & Overcast \\
\hline & TEST-B & SKM & 0930 & 1.6 & --- & 27119 & 767 & 283.4 & 28 & Overcast \\
\hline & TEST-C & SKM & 0930 & 1.5 & --- & 27070 & 766 & 282.6 & 28.5 & Overcast \\
\hline \multirow{3}{*}{ 3-Apr-12 } & TEST-A & SKM & 0900 & --- & 710 & --- & --- & --- & --- & Partly Cloudy \\
\hline & TEST-B & SKM & 0900 & 1.6 & --- & 29368 & 831 & 306.9 & 29 & Partly Cloudy \\
\hline & TEST-C & SKM & 0900 & 1.5 & --- & 29321 & 830 & 306.1 & 28.2 & Partly Cloudy \\
\hline \multirow{3}{*}{ 4-Apr-12 } & TEST-A & SKM & 0900 & --- & 771 & --- & --- & --- & --- & Cloudy \\
\hline & TEST-B & SKM & 0935 & 1.6 & --- & 31726 & 898 & 331.6 & 27 & Cloudy \\
\hline & TEST-C & SKM & 0910 & 1.6 & --- & 31640 & 895 & 330.3 & 27.5 & Cloudy \\
\hline \multirow{3}{*}{ 5-Apr-12 } & TEST-A & SKM & 0745 & --- & 830 & --- & --- & --- & --- & Partly Cloudy \\
\hline & TEST-B & SKM & 0745 & 1.6 & --- & 2094 & 59 & 21.9 & 26.5 & Partly Cloudy \\
\hline & TEST-C & SKM & 0745 & 1.6 & --- & 2127 & 60 & 22.2 & 26.5 & Partly Cloudy \\
\hline \multirow{3}{*}{ 6-Apr-12 } & TEST-A & SKM & 0800 & --- & 894 & --- & --- & --- & --- & Partly Cloudy \\
\hline & TEST-B & SKM & 0800 & 1.6 & --- & 4413 & 125 & 46.1 & 26 & Partly Cloudy \\
\hline & TEST-C & SKM & 0800 & 1.5 & --- & 4453 & 126 & 46.5 & 26 & Partly Cloudy \\
\hline \multirow{3}{*}{ 9-Apr-12 } & TEST-A & SKM & 0745 & --- & 1083 & --- & --- & --- & --- & Overcast \\
\hline & TEST-B & SKM & 0745 & 1.6 & --- & 11274 & 319 & 117.8 & 26.5 & Overcast \\
\hline & TEST-C & SKM & 0745 & 1.6 & --- & 11314 & 320 & 118.2 & 26.5 & Overcast \\
\hline \multirow{3}{*}{ 10-Apr-12 } & TEST-A & SKM & 0727 & --- & 1144.3 & --- & --- & --- & --- & Overcast \\
\hline & TEST-B & SKM & 0727 & 1.5 & --- & 13538 & 383 & 141.5 & 25.5 & Overcast \\
\hline & TEST-C & SKM & 0727 & 1.6 & --- & 13583 & 384 & 141.9 & 25.5 & Overcast \\
\hline \multirow{3}{*}{ 11-Apr-12 } & TEST-A & SKM & 0710 & --- & 1203 & --- & --- & --- & --- & Overcast \\
\hline & TEST-B & SKM & 0710 & 1.6 & --- & 15804 & 447 & 165.1 & 25.5 & Overcast \\
\hline & TEST-C & SKM & 0710 & 1.6 & --- & 15848 & 448 & 165.5 & 25.5 & Overcast \\
\hline \multirow{3}{*}{ 12-Apr-12 } & TEST-A & SKM & 0730 & --- & 1265 & --- & --- & --- & --- & Sunny \\
\hline & TEST-B & SKM & 0730 & 1.6 & --- & 18136 & 513 & 189.5 & 26 & Sunny \\
\hline & TEST-C & SKM & 0730 & 1.6 & --- & 18181 & 515 & 189.9 & 26 & Sunny \\
\hline \multirow{3}{*}{ 13-Apr-12 } & TEST-A & SKM & 0745 & --- & 1327 & --- & --- & --- & --- & Sunny \\
\hline & TEST-B & SKM & 0745 & 1.5 & --- & 20467 & 579 & 213.9 & 27 & Sunny \\
\hline & TEST-C & SKM & 0745 & (4) & --- & (4) & (4) & (4) & 28 & Sunny \\
\hline \multirow{3}{*}{ 16-Apr-12 } & TEST-A & SKM & 0750 & --- & 1511 & --- & --- & --- & --- & Cloudy \\
\hline & TEST-B & SKM & 0750 & 1.6 & --- & 27347 & 774 & 285.7 & 25.5 & Cloudy \\
\hline & TEST-C & SKM & 0750 & (4) & --- & (4) & (4) & (4) & 28.5 & Cloudy \\
\hline \multirow{3}{*}{ 17-Apr-12 } & TEST-A & SKM & 0730 & --- & 1572 & --- & --- & --- & --- & Sunny \\
\hline & TEST-B & SKM & 0730 & 1.6 & --- & 29608 & 838 & 309.4 & 26 & Sunny \\
\hline & TEST-C & SKM & 0730 & (4) & --- & (4) & 0 & (4) & 28.5 & Sunny \\
\hline \multirow{3}{*}{ 18-Apr-12 } & TEST-A & SKM & 0845 & --- & 1637 & --- & --- & --- & --- & Cloudy \\
\hline & TEST-B & SKM & 0855 & 1.6 & --- & 32039 & 907 & 334.8 & 28.5 & Cloudy \\
\hline & TEST-D ${ }^{(4)}$ & SKM & 1115 & 1.6 & --- & 0 & 0 & 0 & 26.5 & Cloudy \\
\hline \multirow{3}{*}{ 19-Apr-12 } & TEST-A & SKM & 0730 & --- & 1695 & --- & --- & --- & --- & Sunny \\
\hline & TEST-B & SKM & 0730 & 1.6 & --- & 2122 & 60 & 22.2 & 26.5 & Sunny \\
\hline & TEST-D & SKM & 0730 & 1.6 & --- & 1926 & 55 & 20.1 & 26 & Sunny \\
\hline \multirow{3}{*}{ 20-Apr-12 } & TEST-A & FG & 0730 & --- & 1755 & --- & --- & --- & --- & Partly Cloudy \\
\hline & TEST-B & FG & 0730 & 1.5 & --- & 4408 & 125 & 46 & 26 & Partly Cloudy \\
\hline & TEST-D & FG & 0730 & 1.6 & --- & 4219 & 119 & 44 & 26 & Partly Cloudy \\
\hline
\end{tabular}




\begin{tabular}{|c|c|c|c|c|c|c|c|c|c|c|}
\hline Date & $\begin{array}{c}\text { Monitoring } \\
\text { System } \\
\end{array}$ & RPT & Time & $\begin{array}{l}\text { Flow } \\
\text { Rate } \\
\text { (cfm) } \\
\end{array}$ & $\begin{array}{c}\text { End } \\
\text { Volume } \\
\left(\mathbf{m}^{3}\right) \\
\end{array}$ & $\begin{array}{c}\text { Total } \\
\text { Flow } \\
\left(\mathbf{f t t}^{3}\right) \\
\end{array}$ & $\begin{array}{c}\text { Total } \\
\text { Flow }\left(\mathbf{f t}^{3}\right) \\
\text { converted } \\
\text { to } \mathbf{~ m}^{3} \\
\end{array}$ & $\begin{array}{c}\text { Elapsed } \\
\text { Time } \\
\text { (Hrs) }\end{array}$ & $\begin{array}{c}\text { Battery } \\
\text { Condition } \\
\text { (V) }\end{array}$ & $\begin{array}{c}\text { Environmental } \\
\text { Conditions }\end{array}$ \\
\hline \multirow{3}{*}{ 23-Apr-12 } & TEST-A & SKM & 0745 & --- & 1935 & --- & --- & --- & --- & Sunny \\
\hline & TEST-B & SKM & 0745 & 1.5 & --- & 11324 & 320 & 118 & 26.5 & Sunny \\
\hline & TEST-D & SKM & 0745 & 1.6 & --- & 11144 & 315 & 116.3 & 26 & Sunny \\
\hline \multirow{3}{*}{ 24-Apr-12 } & TEST-A & SKM & 0730 & --- & 1994 & --- & --- & --- & --- & Sunny \\
\hline & TEST-B & SKM & 0730 & 1.6 & --- & 13609 & 385 & 142.2 & 26.5 & Sunny \\
\hline & TEST-D & SKM & 0730 & 1.6 & --- & 13433 & 380 & 140.1 & 26 & Sunny \\
\hline \multirow{3}{*}{ 25-Apr-12 } & TEST-A & SKM & 0815 & --- & 2056 & --- & --- & --- & --- & Cloudy \\
\hline & TEST-B & SKM & 0815 & 1.6 & --- & 15977 & 452 & 166.9 & 26.5 & Cloudy \\
\hline & TEST-D & SKM & 0815 & 1.6 & --- & 15806 & 447 & 164.9 & 26.5 & Cloudy \\
\hline \multirow{3}{*}{ 26-Apr-12 } & TEST-A & SKM & 0800 & --- & 2115 & --- & --- & --- & --- & Rain \\
\hline & TEST-B & SKM & 0800 & 1.6 & --- & 18235 & 516 & 190.5 & 25 & Rain \\
\hline & TEST-D & SKM & 0800 & 1.6 & --- & 18069 & 511 & 188.5 & 25 & Rain \\
\hline \multirow{3}{*}{ 27-Apr-12 } & TEST-A & FG & 0715 & --- & 2175 & --- & --- & --- & --- & Sunny \\
\hline & TEST-B & FG & 0715 & 1.6 & --- & 20458 & 579 & 213.7 & 26 & Sunny \\
\hline & TEST-D & FG & 0715 & 1.6 & --- & 20298 & 574 & 211.7 & 26 & Sunny \\
\hline \multirow{3}{*}{ 30-Apr-12 } & TEST-A & FG & 0800 & --- & 2360 & --- & --- & --- & --- & Sunny \\
\hline & TEST-B & FG & 0800 & 1.5 & --- & 27424 & 776 & 286.5 & 27.5 & Sunny \\
\hline & TEST-D & FG & 0800 & 1.6 & --- & 27270 & 772 & 284.5 & 27 & Sunny \\
\hline \multirow{3}{*}{ 1-May-12 } & TEST-A & DLM & 0836 & --- & 2423 & --- & --- & --- & --- & Sunny \\
\hline & TEST-B & DLM & 0836 & 1.6 & --- & 29769 & 842 & 311 & 28 & Sunny \\
\hline & TEST-D & DLM & 0836 & 1.6 & --- & 29623 & 838 & 309 & 28 & Sunny \\
\hline \multirow{3}{*}{ 2-May-12 } & TEST-A & SKM & 0810 & --- & 2483 & --- & --- & --- & --- & Sunny \\
\hline & TEST-B & SKM & 0840 & 1.6 & --- & 32058 & 907 & 334.9 & 28.5 & Sunny \\
\hline & TEST-D & SKM & 0825 & 1.6 & --- & 31891 & 903 & 332.7 & 28 & Sunny \\
\hline \multirow{3}{*}{ 3-May-12 } & TEST-A & DLM & 0740 & --- & 2543 & --- & --- & --- & --- & Overcast \\
\hline & TEST-B & DLM & 0740 & & --- & 2197 & 62 & 23 & 26 & Overcast \\
\hline & TEST-D & DLM & 0740 & 1.5 & --- & 2221 & 63 & 23.2 & 23.5 & Overcast \\
\hline \multirow{3}{*}{ 4-May-12 } & TEST-A & DLM & 0725 & --- & 2604 & --- & --- & --- & --- & Overcast \\
\hline & TEST-B & DLM & 0725 & 1.6 & --- & 4469 & 126 & 46.7 & 27 & Overcast \\
\hline & TEST-D & DLM & 0725 & 1.6 & --- & 4496 & 127 & 46.9 & 27 & Overcast \\
\hline \multirow{3}{*}{ 7-May-12 } & TEST-A & SKM & 0740 & --- & 2796 & --- & --- & --- & --- & Sunny \\
\hline & TEST-B & SKM & 0740 & 1.6 & --- & 11378 & 322 & 118.9 & 27 & Sunny \\
\hline & TEST-D & SKM & 0740 & 1.6 & --- & 11416 & 323 & 119.1 & 26.5 & Sunny \\
\hline \multirow{3}{*}{ 8-May-12 } & TEST-A & DLM & 0740 & --- & 2858 & --- & --- & --- & --- & Sunny \\
\hline & TEST-B & DLM & 0740 & 1.6 & --- & 13667 & 387 & 142.8 & 27 & Sunny \\
\hline & TEST-D & DLM & 0740 & 1.6 & --- & 13733 & 389 & 143.1 & 27 & Sunny \\
\hline \multirow{3}{*}{ 9-May-12 } & TEST-A & DLM & 0807 & --- & 2921 & --- & --- & --- & --- & Sunny \\
\hline & TEST-B & DLM & 0807 & 1.6 & --- & 16008 & 453 & 167.3 & 28.5 & Sunny \\
\hline & TEST-D & DLM & 0807 & 1.6 & --- & 16052 & 454 & 167.5 & 27.5 & Sunny \\
\hline \multirow{3}{*}{ 10-May-12 } & TEST-A & DLM & 0800 & --- & 2985 & --- & --- & --- & --- & Sunny \\
\hline & TEST-B & DLM & 0800 & 1.6 & --- & 18287 & 518 & 191.1 & 28.5 & Sunny \\
\hline & TEST-D & DLM & 0800 & 1.6 & --- & 18335 & 519 & 191.3 & 27 & Sunny \\
\hline \multirow{3}{*}{ 11-May-12 } & TEST-A & DLM & 0730 & --- & 3047 & --- & --- & --- & --- & Sunny \\
\hline & TEST-B & DLM & 0730 & 1.6 & --- & 20575 & 582 & 215 & 27.5 & Sunny \\
\hline & TEST-D & DLM & 0730 & 1.6 & --- & 20625 & 584 & 215.2 & 26.5 & Sunny \\
\hline
\end{tabular}




\begin{tabular}{|c|c|c|c|c|c|c|c|c|c|c|}
\hline Date & $\begin{array}{c}\text { Monitoring } \\
\text { System }\end{array}$ & RPT & Time & $\begin{array}{l}\text { Flow } \\
\text { Rate } \\
(\mathbf{c f m}) \\
\end{array}$ & $\begin{array}{c}\text { End } \\
\text { Volume } \\
\left(\mathbf{m}^{3}\right) \\
\end{array}$ & $\begin{array}{c}\text { Total } \\
\text { Flow } \\
\left(\mathbf{f t t}^{3}\right) \\
\end{array}$ & $\begin{array}{c}\text { Total } \\
\text { Flow }\left(\mathbf{f t}^{3}\right) \\
\text { converted } \\
\text { to } \mathbf{~ m}^{3} \\
\end{array}$ & $\begin{array}{c}\text { Elapsed } \\
\text { Time } \\
\text { (Hrs) }\end{array}$ & $\begin{array}{c}\text { Battery } \\
\text { Condition } \\
\text { (V) }\end{array}$ & $\begin{array}{c}\text { Environmental } \\
\text { Conditions }\end{array}$ \\
\hline \multirow{3}{*}{ 14-Мay-12 } & TEST-A & SKM & 0745 & --- & 3230 & -- & --- & --- & -- & Sunny \\
\hline & TEST-B & SKM & 0745 & 1.6 & --- & 27453 & 777 & 286.9 & 24.5 & Sunny \\
\hline & TEST-D & SKM & 0745 & 1.6 & --- & 27515 & 779 & 287.1 & 26.5 & Sunny \\
\hline \multirow{3}{*}{ 15-May-12 } & TEST-A & DLM & 0745 & --- & 3293 & --- & --- & --- & --- & Sunny \\
\hline & TEST-B & DLM & 0745 & 1.5 & --- & 29740 & 842 & 310.8 & 24.5 & Sunny \\
\hline & TEST-D & DLM & 0745 & 1.5 & --- & 29810 & 844 & 311.1 & 26.5 & Sunny \\
\hline \multirow{3}{*}{ 16-May-12 } & TEST-A & DLM & 0730 & --- & 3353 & --- & --- & --- & --- & Sunny \\
\hline & TEST-B & DLM & 0730 & 1.6 & --- & 32012 & 906 & 334.5 & 24 & Sunny \\
\hline & TEST-D & DLM & 0730 & 1.6 & --- & 32082 & 908 & 334.7 & 26 & Sunny \\
\hline \multirow{3}{*}{ 17-May-12 } & TEST-A & DLM & 0805 & --- & 3417.2 & --- & --- & --- & --- & Sunny \\
\hline & TEST-B & DLM & 0805 & 1.6 & --- & 2236 & 63 & 23.3 & 23 & Sunny \\
\hline & TEST-D & DLM & 0805 & 1.6 & --- & 2261 & 64 & 23.6 & 27 & Sunny \\
\hline \multirow{3}{*}{ 18-Маy-12 } & TEST-A & DLM & 0725 & --- & 3478.1 & --- & --- & --- & --- & Sunny \\
\hline & TEST-B & DLM & --- & (5) & --- & (5) & (5) & (5) & 23 & Sunny \\
\hline & TEST-D & DLM & 0725 & 1.5 & --- & 4501 & 127 & 47 & 26 & Sunny \\
\hline \multirow{3}{*}{ 21-May-12 } & TEST-A & SKM & 0750 & --- & 3665 & --- & --- & --- & --- & Cloudy \\
\hline & TEST-B & SKM & 0750 & 1.6 & --- & 10192 & 288 & 106.5 & 25.5 & Cloudy \\
\hline & TEST-D & SKM & 0750 & 1.6 & --- & 11436 & 324 & 119.3 & 26 & Cloudy \\
\hline \multirow{3}{*}{ 22-May-12 } & TEST-A & DLM & 0810 & --- & 3729.7 & --- & --- & --- & --- & Overcast \\
\hline & TEST-B & DLM & 0810 & 1.5 & --- & 12511 & 354 & 130.7 & 26 & Overcast \\
\hline & TEST-D & DLM & 0810 & 1.6 & --- & 13761 & 389 & 143.6 & 27 & Overcast \\
\hline \multirow{3}{*}{ 23-May-12 } & TEST-A & DLM & 0900 & --- & 3795 & --- & --- & --- & --- & Thin Overcast \\
\hline & TEST-B & DLM & 0900 & 1.6 & --- & 14894 & 422 & 155.6 & 27.5 & Thin Overcast \\
\hline & TEST-D & DLM & 0900 & 1.6 & --- & 16147 & 457 & 168.5 & 28 & Thin Overcast \\
\hline \multirow{3}{*}{ 24-May-12 } & TEST-A & SKM & 0900 & --- & 3856 & --- & --- & --- & --- & Sunny \\
\hline & TEST-B & SKM & 0900 & 1.6 & --- & 17198 & 487 & 179.7 & 25 & Sunny \\
\hline & TEST-D & SKM & 0900 & 1.6 & --- & 18451 & 522 & 192.5 & 27.5 & Sunny \\
\hline \multirow{3}{*}{ 25-May-12 } & TEST-A & SKM & 0900 & --- & 3919 & --- & --- & --- & --- & Sunny \\
\hline & TEST-B & SKM & 0900 & 1.6 & --- & 19495 & 552 & 203.7 & 28 & Sunny \\
\hline & TEST-D & SKM & 0900 & 1.6 & --- & 20751 & 587 & 216.5 & 28 & Sunny \\
\hline \multirow{3}{*}{ 29-May-12 } & TEST-A & DLM & 0810 & --- & 4163 & --- & --- & --- & --- & Thin Overcast \\
\hline & TEST-B & DLM & 0810 & 1.5 & --- & 28578 & 809 & 298.6 & 25.5 & Thin Overcast \\
\hline & TEST-D & DLM & 0810 & 1.6 & --- & 29853 & 845 & 311.5 & 28 & Thin Overcast \\
\hline \multirow{3}{*}{ 30-May-12 } & TEST-A & DLM & 0855 & --- & 4227 & --- & --- & --- & --- & Overcast \\
\hline & TEST-B & DLM & 0855 & 1.6 & --- & 30945 & 876 & 323.3 & 24.5 & Overcast \\
\hline & TEST-D & DLM & 0855 & 1.5 & --- & 32219 & 912 & 336.2 & 28 & Overcast \\
\hline \multirow{3}{*}{ 31-May-12 } & TEST-A & DLM & 0825 & --- & 4294 & --- & --- & --- & --- & Cloudy \\
\hline & TEST-B & DLM & 0825 & 1.6 & --- & 2144 & 61 & 22.4 & 24.5 & Cloudy \\
\hline & TEST-D & DLM & 0825 & 1.6 & --- & 2171 & 61 & 22.6 & 26.5 & Cloudy \\
\hline \multirow{3}{*}{ 1-Jun-12 } & TEST-A & DLM & 0750 & --- & 4361 & --- & --- & --- & --- & Cloudy \\
\hline & TEST-B & DLM & 0750 & 1.6 & --- & 4386 & 124 & 45.8 & 24 & Cloudy \\
\hline & TEST-D & DLM & 0750 & 1.5 & --- & 4417 & 125 & 46.1 & 27 & Cloudy \\
\hline \multirow{3}{*}{ 4-Jun-12 } & TEST-A & SKM & 0800 & --- & 4566 & --- & --- & --- & --- & Cloudy \\
\hline & TEST-B & SKM & 0800 & (6) & --- & (6) & (6) & (6) & 23 & Cloudy \\
\hline & TEST-D & SKM & 0800 & 1.6 & --- & 11321 & 320 & 118.1 & 26 & Cloudy \\
\hline
\end{tabular}




\begin{tabular}{|c|c|c|c|c|c|c|c|c|c|c|}
\hline Date & $\begin{array}{c}\text { Monitoring } \\
\text { System }\end{array}$ & RPT & Time & $\begin{array}{l}\text { Flow } \\
\text { Rate } \\
(\mathbf{c f m}) \\
\end{array}$ & $\begin{array}{c}\text { End } \\
\text { Volume } \\
\left(\mathbf{m}^{3}\right) \\
\end{array}$ & $\begin{array}{c}\text { Total } \\
\text { Flow } \\
\left(\mathbf{f t t}^{3}\right) \\
\end{array}$ & $\begin{array}{c}\text { Total } \\
\text { Flow }\left(\mathbf{f t}^{3}\right) \\
\text { converted } \\
\text { to } \mathbf{~ m}^{3} \\
\end{array}$ & $\begin{array}{c}\text { Elapsed } \\
\text { Time } \\
\text { (Hrs) }\end{array}$ & $\begin{array}{c}\text { Battery } \\
\text { Condition } \\
\text { (V) }\end{array}$ & $\begin{array}{c}\text { Environmental } \\
\text { Conditions }\end{array}$ \\
\hline \multirow{3}{*}{ 5-Jun-12 } & TEST-A & FG & 0800 & --- & 4634 & --- & --- & --- & --- & Raining \\
\hline & TEST-B & FG & 0800 & (6) & --- & (6) & (6) & (6) & $(6)$ & Raining \\
\hline & TEST-D & FG & 0800 & 1.6 & --- & 13625 & 386 & 142.1 & 26 & Raining \\
\hline \multirow{3}{*}{ 6-Jun-12 } & TEST-A & FG & 0830 & --- & 4703 & --- & --- & --- & --- & Sunny \\
\hline & TEST-B & FG & 0830 & (6) & --- & (6) & (6) & (6) & (6) & Sunny \\
\hline & TEST-D & FG & 0830 & 1.5 & --- & 15969 & 452 & 166.6 & 28 & Sunny \\
\hline \multirow{3}{*}{ 7-Jun-12 } & TEST-A & FG & 0730 & --- & 4769 & --- & --- & --- & --- & Cloudy \\
\hline & TEST-B & FG & 0730 & (6) & --- & (6) & (6) & (6) & (6) & Cloudy \\
\hline & TEST-D & FG & 0730 & 1.6 & --- & 18193 & 515 & 189.8 & 27 & Cloudy \\
\hline \multirow{3}{*}{ 8-Jun-12 } & TEST-A & DLM & 1315 & --- & 4852 & --- & --- & --- & --- & Partly Cloudy \\
\hline & TEST-B & DLM & 1315 & 1.6 & --- & 13399 & 379 & 140 & 28 & Partly Cloudy \\
\hline & TEST-D & DLM & 1315 & 1.6 & --- & 21029 & 595 & 219.3 & 26 & Partly Cloudy \\
\hline \multirow{3}{*}{ 11-Jun-12 } & TEST-A & FG & 0930 & --- & 5049 & --- & --- & --- & --- & Partly Cloudy \\
\hline & TEST-B & FG & 0830 & (6) & --- & (6) & (6) & (6) & (6) & Partly Cloudy \\
\hline & TEST-D & FG & 0830 & (6) & --- & (6) & (6) & (6) & (6) & Partly Cloudy \\
\hline \multirow{3}{*}{ 12-Jun-12 } & TEST-A & DLM & 1510 & --- & 5130 & --- & --- & --- & --- & Mostly Cloudy \\
\hline & TEST-B & DLM & 1330 & 1.6 & --- & 19866 & 562 & 207.6 & 28 & Mostly Cloudy \\
\hline & TEST-D & DLM & 1500 & 1.5 & --- & 27627 & 782 & 288.2 & 26 & Mostly Cloudy \\
\hline \multirow{3}{*}{ 13-Jun-12 } & TEST-A & DLM & 1100 & --- & 5187 & --- & --- & --- & --- & Clear \\
\hline & TEST-B & DLM & 1000 & 1.6 & --- & 21824 & 618 & 228 & 28 & Clear \\
\hline & TEST-D & DLM & 0910 & 1.6 & --- & 29364 & 831 & 306.3 & 28 & Clear \\
\hline \multirow{3}{*}{ 14-Jun-12 } & TEST-A & DLM & 1111 & --- & 5256 & --- & --- & --- & --- & Sunny \\
\hline & TEST-B & DLM & 1057 & 1.6 & --- & 2370 & 67 & 24.8 & 27 & Sunny \\
\hline & TEST-D & DLM & 1016 & 1.6 & --- & 2385 & 67 & 24.9 & 26.5 & Sunny \\
\hline \multirow{3}{*}{ 15-Jun-12 } & TEST-A & FG & 0830 & --- & 5317 & --- & --- & --- & --- & Sunny \\
\hline & TEST-B & FG & 0900 & 1.6 & --- & 4472 & 127 & 46.8 & 27 & Sunny \\
\hline & TEST-D & FG & 0845 & 1.5 & --- & 4544 & 129 & 47.4 & 28 & Sunny \\
\hline \multirow{3}{*}{ 18-Jun-12 } & TEST-A & SKM & 0750 & --- & 5520 & --- & --- & --- & --- & Cloudy \\
\hline & TEST-B & SKM & 0800 & 1.6 & --- & 11276 & 319 & 117.8 & 28 & Cloudy \\
\hline & TEST-D & SKM & 0755 & 1.6 & --- & 11361 & 322 & 118.5 & 26.5 & Cloudy \\
\hline \multirow{3}{*}{ 19-Jun-12 } & TEST-A & FG & 0830 & --- & 5592 & --- & --- & --- & --- & Cloudy \\
\hline & TEST-B & FG & 0800 & 1.5 & --- & 13620 & 385 & 142.3 & 28 & Cloudy \\
\hline & TEST-D & FG & 0800 & 1.6 & --- & 13708 & 388 & 143 & 28 & Cloudy \\
\hline \multirow{3}{*}{ 20-Jun-12 } & TEST-A & FG & 1300 & --- & 5672 & --- & --- & --- & --- & Sunny \\
\hline & TEST-B & FG & 1315 & 1.6 & --- & 16341 & 462 & 170.8 & 26 & Sunny \\
\hline & TEST-D & FG & 1300 & 1.6 & --- & 16414 & 465 & 171.2 & 26 & Sunny \\
\hline \multirow{3}{*}{ 21-Jun-12 } & TEST-A & FG & 0800 & --- & 5728 & --- & --- & --- & --- & Sunny \\
\hline & TEST-B & FG & 0830 & 1.6 & --- & 18175 & 514 & 189.9 & 27.5 & Sunny \\
\hline & TEST-D & FG & 0815 & 1.6 & --- & 18257 & 517 & 190.5 & 26.5 & Sunny \\
\hline \multirow{3}{*}{ 22-Jun-12 } & TEST-A & DLM & 0753 & --- & 5794 & --- & --- & --- & --- & Overcast \\
\hline & TEST-B & DLM & 0806 & 1.5 & --- & 20448 & 579 & 213.7 & 26.5 & Overcast \\
\hline & TEST-D & DLM & 0801 & 1.6 & --- & 20540 & 581 & 214.3 & 26 & Overcast \\
\hline \multirow{3}{*}{ 25-Jun-12 } & TEST-A & FG & 0915 & --- & 6004 & --- & --- & --- & --- & Overcast \\
\hline & TEST-B & FG & 0900 & 1.5 & --- & 27370 & 775 & 266 & 28 & Overcast \\
\hline & TEST-D & FG & 0930 & 1.6 & --- & 27568 & 780 & 287.6 & 28 & Overcast \\
\hline
\end{tabular}




\begin{tabular}{|c|c|c|c|c|c|c|c|c|c|c|}
\hline Date & $\begin{array}{c}\text { Monitoring } \\
\text { System }\end{array}$ & RPT & Time & $\begin{array}{l}\text { Flow } \\
\text { Rate } \\
(\mathbf{c f m}) \\
\end{array}$ & $\begin{array}{c}\text { End } \\
\text { Volume } \\
\left(\mathbf{m}^{3}\right) \\
\end{array}$ & $\begin{array}{c}\text { Total } \\
\text { Flow } \\
\left(\mathbf{f t}^{3}\right) \\
\end{array}$ & $\begin{array}{c}\text { Total } \\
\text { Flow }\left(\mathbf{f t}^{3}\right) \\
\text { converted } \\
\text { to } \mathbf{~ m}^{3} \\
\end{array}$ & $\begin{array}{c}\text { Elapsed } \\
\text { Time } \\
\text { (Hrs) } \\
\end{array}$ & $\begin{array}{c}\text { Battery } \\
\text { Condition } \\
\text { (V) }\end{array}$ & $\begin{array}{c}\text { Environmental } \\
\text { Conditions } \\
\end{array}$ \\
\hline \multirow{3}{*}{ 26-Jun-12 } & TEST-A & FG & 1100 & --- & 6079 & -- & --- & --- & -- & Raining \\
\hline & TEST-B & FG & 1130 & 1.6 & --- & 29894 & 846 & 312.3 & 28 & Raining \\
\hline & TEST-D & FG & 1115 & 1.6 & --- & 30081 & 851 & 313.8 & 28 & Raining \\
\hline \multirow{3}{*}{ 27-Jun-12 } & TEST-A & FG & 1345 & --- & 6155 & --- & --- & --- & --- & Sunny \\
\hline & TEST-B & FG & 1430 & 1.6 & --- & 32335 & 915 & 337.8 & 26 & Sunny \\
\hline & TEST-D & FG & 1400 & 1.6 & --- & 32598 & 923 & 340.1 & 26 & Sunny \\
\hline \multicolumn{11}{|c|}{$\begin{array}{l}\text { (1) This data is dependent on the type of monitoring system (120-V versus 24-V) and is not available. } \\
\text { (2) Data not collected. } \\
\text { (3) Increased set flow to } 1.6 \text {. } \\
\text { (4) System failed and 4-in. filter taken out of service; sent HI-Q blower unit back to vendor for repair. TEST-C was } \\
\text { replaced with TEST-D. TEST-C was a 4-in. filter, and TEST-D is a 2-in. filter. } \\
\text { (5) System failed then started unexpectedly after being inoperative for } 13 \text { hours. } \\
\text { (6) System power controller failed. System off line. }\end{array}$} \\
\hline
\end{tabular}





\section{Appendix B}

\section{GEL Laboratories Analytical Results for 2-in Sample Filters}





\section{Appendix B}

\section{Analytical Results for 2-in Sample Filters}

\begin{tabular}{|c|c|c|c|c|c|c|}
\hline Radionuclide & $\begin{array}{c}\text { Monitoring } \\
\text { System }\end{array}$ & Sample On & Sample Off & $\begin{array}{c}\text { Lab } \\
\text { Qualifier }\end{array}$ & $\begin{array}{c}\text { Value } \\
\text { Reported } \\
\text { (pCi/m3) }\end{array}$ & $\begin{array}{c}\text { Error/ Standard } \\
\text { Deviation } \\
\text { (pCi/m3) }\end{array}$ \\
\hline \multirow[t]{24}{*}{ ALPHA } & \multirow[t]{11}{*}{ TEST-A } & 17-Jan-12 & 8-Feb-12 & & $7.66 \mathrm{E}-04$ & 2.78E-04 \\
\hline & & 8-Feb-12 & 22-Feb-12 & & 8.24E-04 & 4.12E-04 \\
\hline & & 22-Feb-12 & 7-Mar-12 & & $8.47 \mathrm{E}-04$ & 3.75E-04 \\
\hline & & 7-Mar-12 & 21-Mar-12 & $\mathrm{U}$ & 2.65E-04 & 2.45E-04 \\
\hline & & 21-Mar-12 & 4-Apr-12 & $\mathrm{U}$ & 3.65E-04 & 2.99E-04 \\
\hline & & 4-Apr-12 & 18-Apr-12 & & 4.85E-04 & 3.03E-04 \\
\hline & & 18-Apr-12 & 2-Маy-12 & U & $3.30 \mathrm{E}-04$ & 2.63E-04 \\
\hline & & 2-May-12 & 16-May-12 & & 5.53E-04 & 2.93E-04 \\
\hline & & 16-Мay-12 & 30-May-12 & & $5.21 \mathrm{E}-04$ & 2.89E-04 \\
\hline & & 30-May-12 & 13-Jun-12 & & 5.25E-04 & 2.76E-04 \\
\hline & & 13-Jun-12 & 27-Jun-12 & & 3.88E-04 & 2.42E-04 \\
\hline & \multirow[t]{8}{*}{ TEST-B } & 22-Feb-12 & 7-Mar-12 & & 5.09E-04 & 2.93E-04 \\
\hline & & 7-Mar-12 & 21-Mar-12 & & 8.95E-04 & 4.07E-04 \\
\hline & & 21-Mar-12 & 4-Apr-12 & & $4.24 \mathrm{E}-04$ & 2.62E-04 \\
\hline & & 4-Apr-12 & 18-Apr-12 & & $4.23 \mathrm{E}-04$ & 2.70E-04 \\
\hline & & 18-Apr-12 & 2-Маy-12 & & 4.05E-04 & 2.57E-04 \\
\hline & & 2-Мау-12 & 16-Мау-12 & & 7.46E-04 & 3.41E-04 \\
\hline & & 16-Мау-12 & 30-May-12 & $\mathrm{U}$ & 1.89E-04 & $2.21 \mathrm{E}-04$ \\
\hline & & 13-Jun-12 & 27-Jun-12 & & 4.93E-04 & 2.99E-04 \\
\hline & \multirow{5}{*}{ TEST-D } & 18-Apr-12 & 2-Мay-12 & & $6.91 \mathrm{E}-04$ & 3.69E-04 \\
\hline & & 2-May-12 & 16-May-12 & & 7.67E-04 & $3.74 \mathrm{E}-04$ \\
\hline & & 16-May-12 & 30-May-12 & & 6.33E-04 & 2.86E-04 \\
\hline & & 30-May-12 & 13-Jun-12 & & 4.44E-04 & $3.26 \mathrm{E}-04$ \\
\hline & & 13-Jun-12 & 27-Jun-12 & & 3.91E-04 & $2.45 \mathrm{E}-04$ \\
\hline \multirow[t]{17}{*}{ BETA } & \multirow[t]{11}{*}{ TEST-A } & 17-Jan-12 & 8-Feb-12 & & $1.75 \mathrm{E}-02$ & $1.09 \mathrm{E}-03$ \\
\hline & & 8-Feb-12 & 22-Feb-12 & & 2.03E-02 & $1.46 \mathrm{E}-03$ \\
\hline & & 22-Feb-12 & 7-Mar-12 & & 1.15E-02 & $1.10 \mathrm{E}-03$ \\
\hline & & 7-Mar-12 & 21-Mar-12 & & $9.74 \mathrm{E}-03$ & 9.67E-04 \\
\hline & & 21-Mar-12 & 4-Apr-12 & & 1.03E-02 & $1.01 \mathrm{E}-03$ \\
\hline & & 4-Apr-12 & 18-Apr-12 & & $1.23 \mathrm{E}-02$ & $1.05 \mathrm{E}-03$ \\
\hline & & 18-Apr-12 & 2-Маy-12 & & $1.12 \mathrm{E}-02$ & $1.01 \mathrm{E}-03$ \\
\hline & & 2-May-12 & 16-May-12 & & 1.59E-02 & $1.22 \mathrm{E}-03$ \\
\hline & & 16-Маy-12 & 30-May-12 & & $1.40 \mathrm{E}-02$ & 1.14E-03 \\
\hline & & 30-May-12 & 13-Jun-12 & & $9.40 \mathrm{E}-03$ & 8.40E-04 \\
\hline & & 13-Jun-12 & 27-Jun-12 & & $9.23 \mathrm{E}-03$ & 8.28E-04 \\
\hline & \multirow[t]{6}{*}{ TEST-B } & 22-Feb-12 & 7-Mar-12 & & $1.06 \mathrm{E}-02$ & 9.69E-04 \\
\hline & & 7-Mar-12 & 21-Mar-12 & & $9.72 \mathrm{E}-03$ & 9.67E-04 \\
\hline & & 21-Mar-12 & 4-Apr-12 & & 9.61E-03 & 8.68E-04 \\
\hline & & 4-Apr-12 & 18-Apr-12 & & $1.07 \mathrm{E}-02$ & 8.91E-04 \\
\hline & & 18-Apr-12 & 2-Мay-12 & & $1.05 \mathrm{E}-02$ & 8.82E-04 \\
\hline & & 2-May-12 & 16-May-12 & & 1.43E-02 & 1.09E-03 \\
\hline
\end{tabular}




\begin{tabular}{|c|c|c|c|c|c|c|}
\hline Radionuclide & $\begin{array}{l}\text { Monitoring } \\
\text { System }\end{array}$ & Sample On & Sample Off & $\begin{array}{c}\text { Lab } \\
\text { Qualifier }\end{array}$ & $\begin{array}{c}\text { Value } \\
\text { Reported } \\
\text { (pCi/m3) }\end{array}$ & $\begin{array}{l}\text { Error/ Standard } \\
\text { Deviation } \\
\text { (pCi/m3) }\end{array}$ \\
\hline \multirow[t]{7}{*}{ BETA } & \multirow[t]{2}{*}{ TEST-B } & 16-Мay-12 & 30-Мay-12 & & $1.24 \mathrm{E}-02$ & $1.23 \mathrm{E}-03$ \\
\hline & & 13-Jun-12 & 27-Jun-12 & & 8.77E-03 & 8.68E-04 \\
\hline & \multirow[t]{5}{*}{ TEST-D } & 18-Apr-12 & 2-Маy-12 & & 1.22E-02 & $9.44 \mathrm{E}-04$ \\
\hline & & 2-May-12 & 16-Мау-12 & & 1.63E-02 & $1.19 \mathrm{E}-03$ \\
\hline & & 16-Маy-12 & 30-Мау-12 & & 1.33E-02 & 1.10E-03 \\
\hline & & 30-Маy-12 & 13-Jun-12 & & $1.11 \mathrm{E}-02$ & $9.51 \mathrm{E}-04$ \\
\hline & & 13-Jun-12 & 27-Jun-12 & & $1.02 \mathrm{E}-02$ & 8.64E-04 \\
\hline \multirow[t]{3}{*}{ Am-241 } & TEST-A & 17-Jan-12 & 27-Jun-12 & $\mathrm{U}$ & $1.25 \mathrm{E}-06$ & 3.70E-06 \\
\hline & TEST-B & 22-Feb-12 & 27-Jun-12 & U & $-8.96 \mathrm{E}-08$ & $2.47 \mathrm{E}-06$ \\
\hline & TEST-D & 18-Apr-12 & 27-Jun-12 & $\mathrm{U}$ & $-2.60 \mathrm{E}-06$ & 5.43E-06 \\
\hline \multirow[t]{3}{*}{ Am-243 } & TEST-A & 17-Jan-12 & 27-Jun-12 & $\mathrm{U}$ & $2.19 \mathrm{E}-06$ & 6.64E-06 \\
\hline & TEST-B & 22-Feb-12 & 27-Jun-12 & U & $-2.08 \mathrm{E}-06$ & 2.91E-06 \\
\hline & TEST-D & 18-Apr-12 & 27-Jun-12 & $\mathrm{U}$ & $-1.14 \mathrm{E}-06$ & 3.88E-06 \\
\hline \multirow[t]{3}{*}{ Cm-243/244 } & TEST-A & 17-Jan-12 & 27-Jun-12 & $\mathrm{U}$ & $-2.08 \mathrm{E}-06$ & $3.55 \mathrm{E}-06$ \\
\hline & TEST-B & 22-Feb-12 & 27-Jun-12 & $\mathrm{U}$ & $-2.26 \mathrm{E}-06$ & 2.72E-06 \\
\hline & TEST-D & 18-Apr-12 & 27-Jun-12 & U & $-5.16 \mathrm{E}-06$ & 4.95E-06 \\
\hline \multirow[t]{4}{*}{ Co-60 } & TEST-A & 17-Jan-12 & 4-Apr-12 & $\mathrm{U}$ & $-3.30 \mathrm{E}-05$ & $6.60 \mathrm{E}-04$ \\
\hline & TEST-A & 4-Apr-12 & 27-Jun-12 & U & $-3.25 E-05$ & 4.99E-04 \\
\hline & TEST-B & 22-Feb-12 & 27-Jun-12 & U & $-9.54 \mathrm{E}-05$ & 3.01E-04 \\
\hline & TEST-D & 18-Apr-12 & 27-Jun-12 & $\mathrm{U}$ & $-9.49 \mathrm{E}-06$ & 6.70E-04 \\
\hline \multirow[t]{3}{*}{$\mathrm{Pu}-238$} & TEST-A & 17-Jan-12 & 27-Jun-12 & $\mathrm{U}$ & $3.85 \mathrm{E}-07$ & $1.85 \mathrm{E}-06$ \\
\hline & TEST-B & 22-Feb-12 & 27-Jun-12 & U & 8.36E-07 & 1.55E-06 \\
\hline & TEST-D & 18-Apr-12 & 27-Jun-12 & $\mathrm{U}$ & $0.00 \mathrm{E}+00$ & 2.03E-06 \\
\hline \multirow[t]{3}{*}{$\mathrm{Pu}-239 / 240$} & TEST-A & 17-Jan-12 & 27-Jun-12 & & $4.61 \mathrm{E}-06$ & $3.12 \mathrm{E}-06$ \\
\hline & TEST-B & 22-Feb-12 & 27-Jun-12 & U & 1.95E-06 & 2.32E-06 \\
\hline & TEST-D & 18-Apr-12 & 27-Jun-12 & & 4.16E-06 & $3.40 \mathrm{E}-06$ \\
\hline \multirow[t]{3}{*}{ U-233/234 } & TEST-A & 17-Jan-12 & 27-Jun-12 & & 4.82E-05 & 1.63E-05 \\
\hline & TEST-B & 22-Feb-12 & 27-Jun-12 & & 4.71E-05 & $1.22 \mathrm{E}-05$ \\
\hline & TEST-D & 18-Apr-12 & 27-Jun-12 & & $4.01 \mathrm{E}-05$ & 1.55E-05 \\
\hline
\end{tabular}


Appendix C

\section{Daily Monitoring Checklist Sample Form}





\section{Appendix C}

\section{Daily Monitoring Checklist Sample Form}

\section{Daily Monitoring Checklist}

\begin{tabular}{|c|c|c|c|c|c|c|c|c|c|}
\hline & Monitor & $\begin{array}{l}\text { RPT } \\
\text { Initials }\end{array}$ & Date & Time & Flow Rate & $\begin{array}{l}\text { Total } \\
\text { Flow }\end{array}$ & $\begin{array}{l}\text { Elapsed } \\
\text { Time }\end{array}$ & $\begin{array}{c}\text { Battery } \\
\text { Condition } \\
\text { (Voltage) }\end{array}$ & $\begin{array}{l}\text { Environmental } \\
\text { Conditions } \\
\text { (cloudy, } \\
\text { sunny, etc.) }\end{array}$ \\
\hline & TEST-A & $D M$ & $6-14$ & $\not 1 / 7 / h$ & $\cdots-N / A \cdots$ & 5256 & $\cdots-N / A \cdots$ & $\cdots--N / A-\cdots$ & \multirow{3}{*}{ Suaky } \\
\hline 2 & TEST-B & & 1 & 1057 & 7.6 & 2370 & 24.8 & 27 & \\
\hline & TEST-D & 1 & $b$ & 10.16 & $i .6$ & 2385 & 24.4 & 26.5 & \\
\hline & TEST-A & Ft & $6-15-12$ & 0830 & $\cdots--N / A \cdots$ & 5317 & $-\cdots-N / A-\cdots$ & $\cdots-N / A \cdots$ & \multirow{3}{*}{ sunny } \\
\hline 2 & TEST-B & 12 & $6-15,12$ & 0900 & 1.6 & 4472 & 46.8 & 27 & \\
\hline 1 & TEST-D & 32 & $6-15-12$ & 0845 & 1.5 & 4544 & 42.4 & 28 & \\
\hline & TEST-A & $56 \mathrm{~cm}$ & $6-18-12$ & 0750 & $\cdots-N / A \cdots$ & 5520 & $\cdots-N / A \cdots$ & $\cdots-\cdots$ & \multirow{3}{*}{ Clovoy } \\
\hline 2 & TEST-B & Sikm & $6-18+12$ & 0800 & 1.6 & 11276 & 117.8 & 28 & \\
\hline 1 & TEST-D & $\mathrm{Sicm}$ & $6-18-12$ & 0755 & 1.6 & 11361 & 118.5 & 26.5 & \\
\hline & TEST-A & D2 & $6 / 9-12$ & 0830 & $---N / A-\cdots$ & 5592 & $\cdots-\cdots$ N/A- - & $-\cdots--N / A-\cdots$ & \multirow{3}{*}{ cloudy } \\
\hline 2 & TEST-B & ts & $6-19-12$ & 0800 & 1.5 & 13620 & 142.3 & 28 & \\
\hline 1 & TEST-D & IA & $6-19.12$ & 0800 & 1.6 & 13708 & 143.0 & 28 & \\
\hline & TEST-A & 122 & $6-2 v-12$ & 1300 & $\cdots---N / A \cdots$ & 5672 & $\cdots--N / A-\cdots$ & $-\cdots-N / A-\cdots$ & \multirow{3}{*}{ Sunny } \\
\hline 2 & TEST-B & T2 & $6.20 \cdot 12$ & 1315 & 1.6 & 16341 & 170.8 & 26 & \\
\hline \multirow{16}{*}{$\begin{array}{l}\frac{\mathrm{T}}{\mathrm{T}} \mathrm{T} \\
\mathrm{T} \\
\mathrm{T} \\
\mathrm{T} \\
\mathrm{T} \\
\mathrm{T} \\
\mathrm{T}\end{array}$} & TEST-D & 12 & 6.2012 & 1300 & 7.6 & 16414 & 171.2 & 26 & \\
\hline & NoT-A & & & & $\cdots-N / A \cdots$ & & ----N/A---- & $----N / A---$ & \\
\hline & & & & & & & & & \\
\hline & TEST-D & & & & & & & & \\
\hline & TEST-A & & & & $\cdots-N / A \cdots$ & & $\cdots-\mathrm{N} /$ & & \\
\hline & TEST-B & & & & & & & & \\
\hline & TEST-D & & & & & & & & \\
\hline & TEST-A & & & & & & $\cdots-\cdots / A \cdots$ & $\cdots--N / A-\cdots$ & \\
\hline & TEST-B & & & & & & & & \\
\hline & TEST-D & & & & & & & & \\
\hline & TEST-A & & & & A--.- & & & $-\cdots-N / A-\cdots$ & \\
\hline & TEST-B & & & & & & & & \\
\hline & TEST-D & & & & & & & & \\
\hline & TEST-A & & & & $\cdots--N / A \cdots$ & & $---\mathrm{N} / \mathrm{A}-\cdots$ & $\cdots--N / A$ & \\
\hline & TEST & & & & & & & & \\
\hline & To1-D & & & & & & & & \\
\hline & Comments & any & al ev & $\mathrm{ts}$ fr & bove (ret & nce Loca & and Date): & & \\
\hline
\end{tabular}








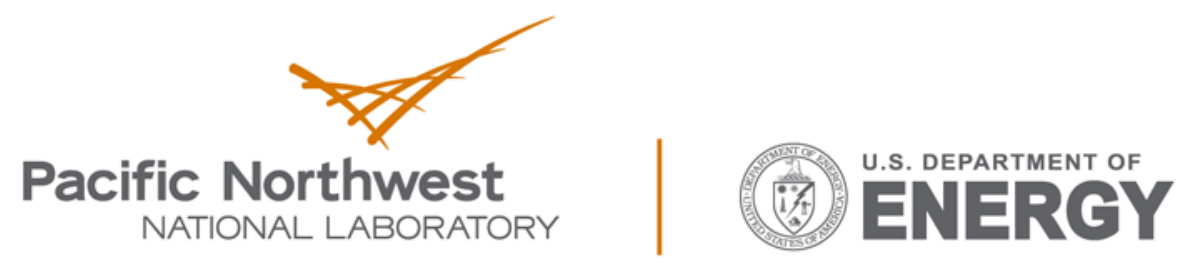

Proudly Operated by Battelle Since 1965

902 Battelle Boulevard

P.O. Box 999

Richland, WA 99352

1-888-375-PNNL (7665)

www.pnnl.gov 